\title{
National Audit of Quality Assurance for Intensity Modulated Radiotherapy and Volumetric Modulated Arc Therapy
}

\section{NEDERLANDSE COMMISSIE VOOR STRALINGSDOSIMETRIE}

Report 28 of the Netherlands Commission on Radiation Dosimetry

March 2018 


\section{Disclaimer regarding NCS reports}

The NCS frequently publishes reports for fellow professionals in which recommendations are given for various quality control procedures or otherwise. The members of the NCS board and the members of the concerning subcommittee do not claim any authority exceeding that of their professional expertise. Responsibility on how the NCS recommendations are implemented lies with the user, taking into account the practice in his/her institution. 


\section{Preface}

The Nederlandse Commissie voor Stralingsdosimetrie (NCS, Netherlands Commission on Radiation Dosimetry, http://www.radiationdosimetry.org) was officially established on 3 September 1982 with the aim of promoting the appropriate use of dosimetry of ionising radiation both for scientific research and practical applications. The NCS is chaired by a board of scientists, installed upon the nomination of the supporting societies, including the Nederlandse Vereniging voor Radiotherapie en Oncologie (Netherlands Society for Radiotherapy and Oncology), the Nederlandse Vereniging voor Nucleaire Geneeskunde (Dutch Society of Nuclear Medicine), the Nederlandse Vereniging voor Klinische Fysica (Dutch Society for Medical Physics), the Nederlandse Vereniging voor Radiobiologie (Netherlands Radiobiological Society), the Nederlandse Vereniging voor Stralingshygiëne (Netherlands Society for Radiological Protection), the Nederlandse Vereniging voor Medische Beeldvorming en Radiotherapie (Dutch Society for Medical Imaging and Radiotherapy), the Nederlandse Vereniging van Klinisch Fysisch Medewerkers (Dutch Society for Medical Physics Engineers), the Nederlandse Vereniging voor Radiologie (Radiological Society of the Netherlands) and the Belgische Vereniging voor Ziekenhuisfysici/Société Belge des Physiciens des Hôpitaux (Belgian Hospital Physicists Association). To pursue its aims, the NCS accomplishes the following tasks: participation in dosimetry standardisation and promotion of dosimetry intercomparisons, drafting of dosimetry protocols, collection and evaluation of physical data related to dosimetry. Furthermore, the commission shall maintain or establish links with national and international organisations concerned with ionising radiation and promulgate information on new developments in the field of radiation dosimetry.

Current members of the board of the NCS

J.B. van de Kamer, Chairman T.W.M. Grimbergen, Vice-Chairman J.A. de Pooter, Secretary J.M.J. Hermans, Treasurer A. Rijnders N. de Graaf F.W. Wittkämper M.K. de Fluiter-Zeeman J.R. de Jong P. Sminia K. Franken 
National audit of Quality Assurance for Intensity Modulated Radiotherapy and Volumetric Modulated Arc Therapy

March 2018

This report was prepared by a subcommittee of the Netherlands Commission on Radiation Dosimetry (NCS).

Members of the subcommittee:

Enrica Seravalli Leo van Battum Marion van Gellekom

Anette Houweling Jochem Kaas Marc Kuik Erik Loeff Jacco de Pooter Thom Raaben Wilfred de Vries

NCS, Delft, The Netherlands

For more information on NCS Reports, see https://radiationdosimetry.org 


\section{Summary}

This audit has been conducted to independently validate quality assurance (QA) methods of patient treatment plans for intensity modulated radiation therapy (IMRT) and volumetric arc therapy (VMAT) delivery techniques used clinically in the Netherlands. To this end, a fixed set of treatment plans was measured in all 21 Dutch radiotherapy centres, on 22 sites.

The treatment plans were created by the audit team, consisting of simple and more complex IMRT and VMAT plans, and one stereotactic VMAT plan. All plans used 6 MV photon beams or arcs for both Varian (Varian Medical Systems, Palo Alto, California) and Elekta (Elekta Instrument $A B$, Stockholm, Sweden) linear accelerators. The plans were imported into the participating institute's treatment planning system for dose computation. The audit team subsequently performed measurements using the audit equipment: an ionisation chamber, radiochromic film and a $2 \mathrm{D}$ ionisation chamber array, all inserted in the same phantom. Additionally, the participating institute performed QA measurements for the same treatment plans using their local equipment according to local procedures. Differences between the dose distributions measured by the audit team and computed by the institute were analysed. In addition, the agreement between the audit and institute QA results was studied.

For the majority of the cases, the results of the audit did meet the acceptance criteria and were in agreement with the institute QA measurements. In only a few cases, results failed to meet the acceptance criteria for both measurements of the audit and the institute. The level of agreement and disagreement between the audit and the institute QA result varied among the audit measurement dosimeters (i.e. ionisation chamber, film or 2D array).

In conclusion, the results of this audit show that in general the QA procedure of IMRT and VMAT plans in the Netherlands is excellent, although a variation in response was observed for the different QA dosimetry methods employed during the audit. Therefore, users should be aware of the limitations of each QA method and when in doubt repeat the measurement with different dosimetry equipment. In general, it is recommended to have an independent validation of QA methods of treatment plans after following major clinical practice modifications in the treatment chain.

This subcommittee had its first meeting on 18 February 2014.

DOI: $\underline{10.25030 / \text { ncs-028 }}$ 


\section{Contents}

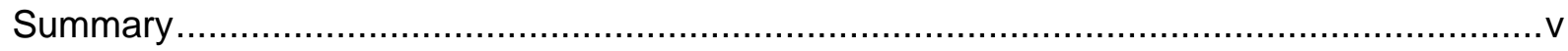

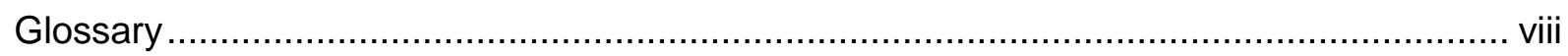

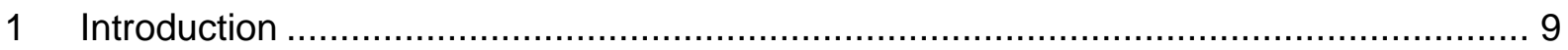

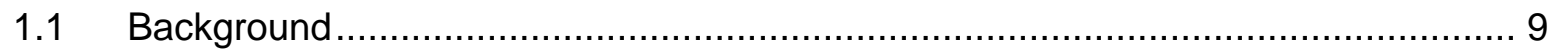

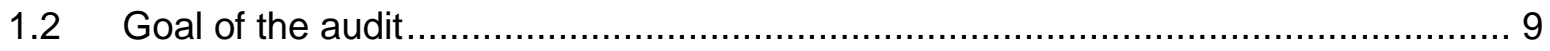

2 Preparation and development of the audit framework .............................................11

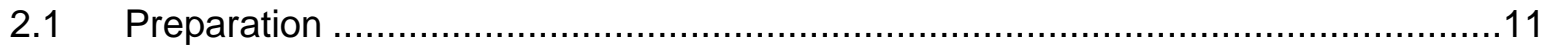

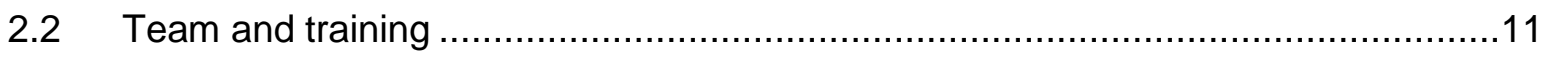

2.3 Measurement protocol................................................................................. 11

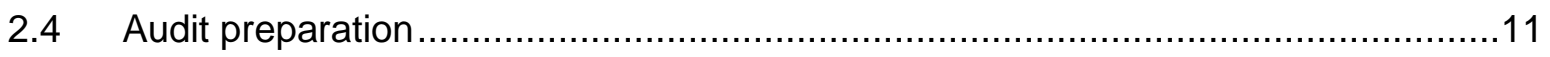

2.5 Scheduling and logistics ........................................................................

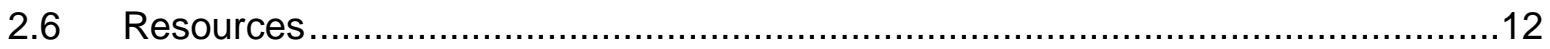

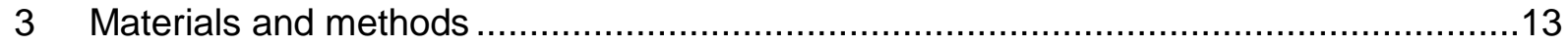

3.1 Available TPS linac combinations in the Netherlands .......................................13

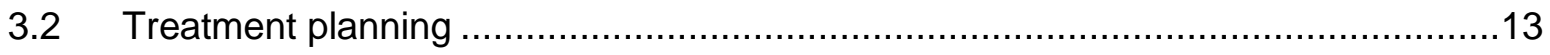

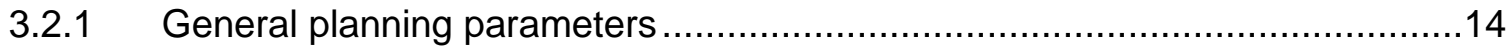

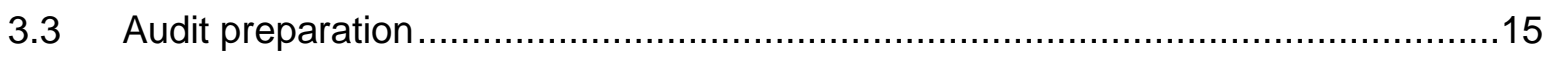

3.3.1 Distribution of audit treatment plans ..................................................... 15

3.3.2 Dose calculation in each institute .......................................................... 16

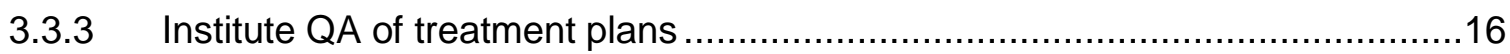

3.4 Audit equipment and measurements methods...............................................17

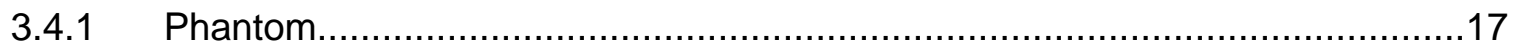

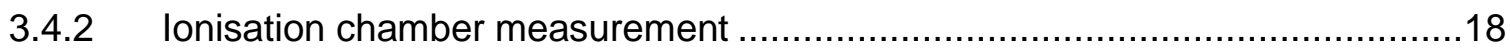

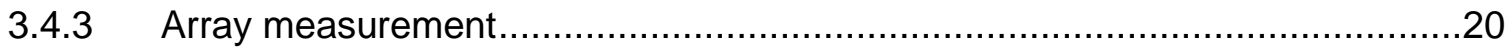

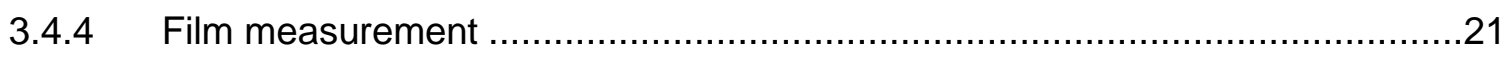

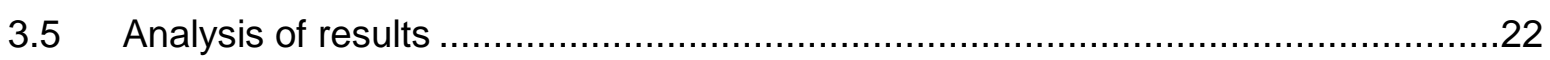

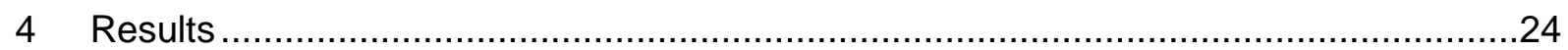

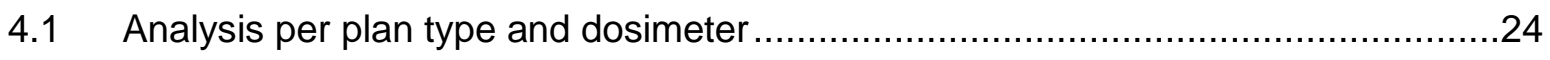

4.1.1 Pass/Fail agreement between audit and institute QA ................................24

4.1.2 Distribution of the audit and institute QA results ........................................26

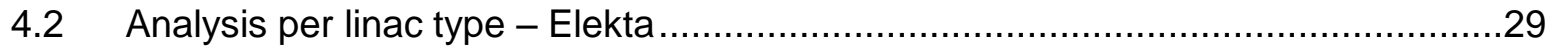

4.2.1 Pass/fail agreement between audit and institute QA results .........................29

4.2.2 Distribution of the audit and institute $Q A$ results ..........................................

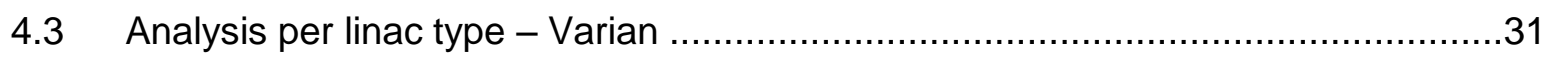


4.3.1 Pass/fail agreement between audit and institute QA results ..........................31

4.3.2 Distribution of the audit and institute QA results ........................................

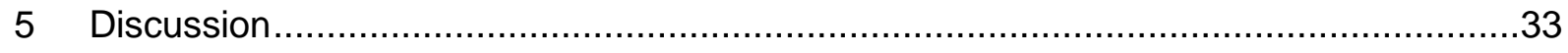

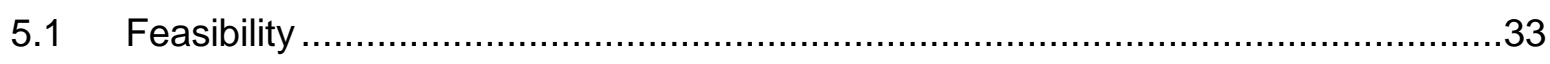

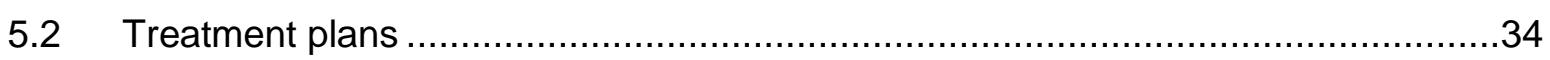

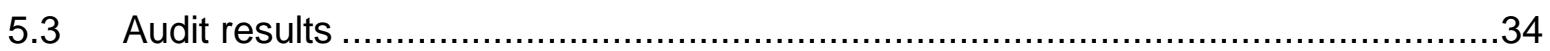

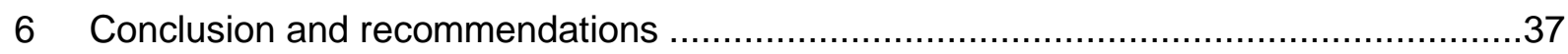

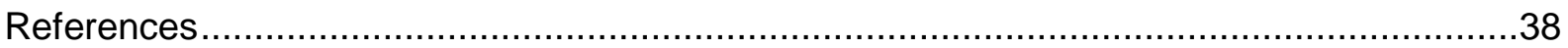

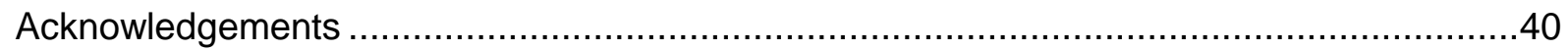

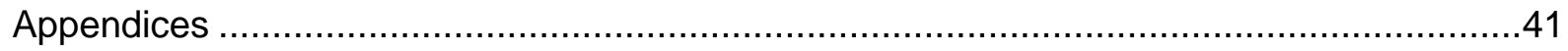

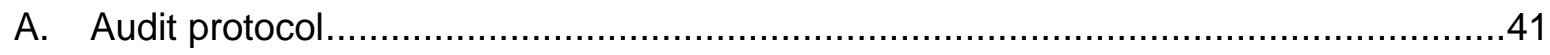

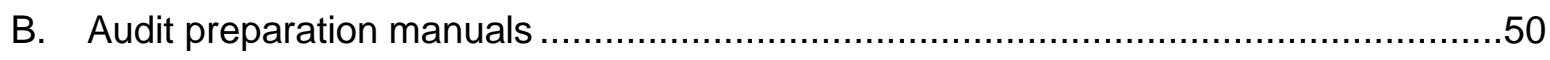

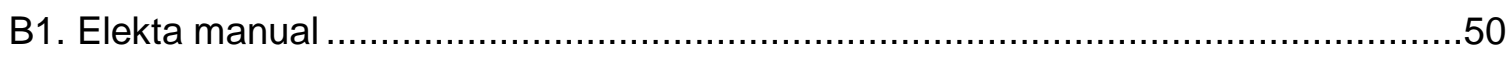

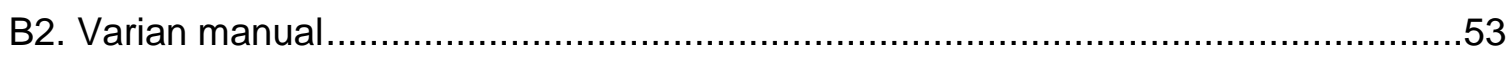

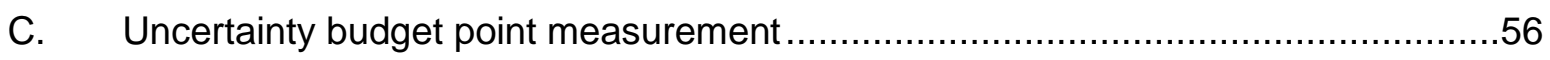

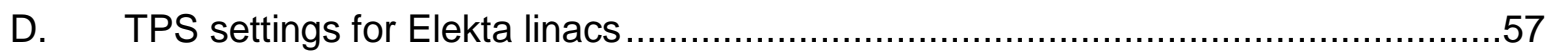




\section{Glossary}

$\begin{array}{ll}\text { CT } & \text { Computer Tomography } \\ \text { DICOM } & \text { Digital Imaging and Communications in Medicine } \\ \text { EPID } & \text { Electronic Portal Imaging Device } \\ \text { HU } & \text { Hounsfield Unit } \\ \text { IMRT } & \text { Intensity-Modulated Radiation Therapy } \\ \text { linac } & \text { Linear accelerator } \\ \text { MLC } & \text { Multi-Leaf Collimator } \\ \text { MU } & \text { Monitor Unit } \\ \text { NCS } & \text { Nederlandse Commissie voor Stralingsdosimetrie } \\ \text { OD } & \text { Optical Density } \\ \text { QA } & \text { Quality Assurance } \\ \text { SSD } & \text { Source to Surface Distance } \\ \text { TPS } & \text { Treatment Planning System } \\ \text { VMAT } & \text { Volumetric Modulated Arc Therapy }\end{array}$




\section{Introduction}

\subsection{Background}

Intensity-modulated radiation therapy (IMRT) and volumetric modulated arc therapy (VMAT) techniques have become standard practice in the Netherlands. Given the complexity of IMRT and VMAT plans, verification is advised to check whether the dose delivery for a specific plan is in accordance with the dose as calculated by the treatment planning system [1,2]. As dose verification of treatment plans is time consuming, the applied methods should not only be accurate but also time efficient.

The Netherlands Commission on Radiation Dosimetry (NCS) has published recommendations on the procedures for the verification of IMRT and VMAT plans $[3,4]$. However, despite these recommendations, local implementation of Quality Assurance (QA) methods and the used measurement equipment may vary considerably. This variation raises questions regarding the accuracy of the treatment plan verification within the Netherlands. It would be useful to know how consistent the results of these verifications are among centres using different measurement equipment and protocols.

Dosimetric audits play a key role in the process of external accreditation of complex radiotherapy techniques and in ensuring the safe and accurate fulfilment of the prescribed dose in the treatment chain $[5,6]$.

Therefore, a validation process was proposed to assess the agreement between dose distribution verification based on local methods and those of an external audit.

\subsection{Goal of the audit}

The purpose of this audit was to independently validate dose distribution measurement methods for IMRT and VMAT plans, used clinically in the Netherlands, employing the same set of treatment plans for all the participating institutes. This in contrast to most national audits.

Using a common set of treatment plans excludes differences due to plan quality or optimisation methods. Simple and complex IMRT and VMAT plans were created by the audit team for both Varian (Varian Medical Systems, Palo Alto, California) and Elekta (Elekta Instrument $A B$, Stockholm, Sweden) linacs.

The set of plans was provided to the participating institutes together with the computed tomography (CT) scan of the audit phantom (Octavius® II, PTW Freiburg GmbH, Freiburg, 
Germany). Each centre imported the CT scan and plans in its own treatment planning system and calculated the dose using its clinical settings. Prior to the audit, each institute was asked to measure the audit plans using its local equipment and QA procedure.

The audit measurements of each plan were performed using three different measurement methods with different characteristics: a PinPoint ionisation chamber for an absolute dose measurements, radiochromic film for a 2D measurement with high spatial resolution and an array of ionisation chambers for a 2D measurement with high reproducibility. The audit results were compared to the QA measurement results performed by each institute.

The audit was performed at the main locations of the 21 Dutch radiotherapy centres and on the satellite location of the South West Radiotherapy Institute having a linac type different from the main location (in total 22 sites). 


\section{Preparation and development of the audit framework}

\subsection{Preparation}

A short questionnaire was sent to all Dutch radiotherapy institutes prior to the audit to gather information about the DICOM import options of the institute's treatment planning system, the preferred type of treatment plans to be verified and details about the QA equipment and procedures. Taking this information into account, the audit was designed by the subcommittee. The design process took approximately half a year. It included creating the measurement and analysis protocols, the treatment plans, as well as organising the audit logistics.

\subsection{Team and training}

The audit measurement team consisted of eleven members including all nine subcommittee members and two additional members to share the workload of the measurements. All members, consisting of medical physics experts and medical physics engineers, were considered competent to act as auditors with sufficient knowledge and experience in the field of radiation dosimetry and QA. During the measurement sessions there were always two auditors present. Prior to the start of the auditing process, several training sessions were organised. The two-fold aim of the training was to familiarise the audit team with the measurement equipment and to test the clarity and feasibility of the measurement protocol.

\subsection{Measurement protocol}

Due to the various advantages and disadvantages of the available QA measurement devices, the subcommittee decided to perform the audit measurements using three different dosimeters: ionisation chamber (absolute dose measurement), 2D ionisation chamber array (2D measurement with high reproducibility) and radiochromic film (2D measurement with high resolution). The measurement protocol (Appendix 1A) was designed and fine-tuned by the audit team over a period of half a year.

\subsection{Audit preparation}

An audit preparation manual was provided to the institutes together with the treatment plans and the CT of the audit phantom (Appendices B1. Elekta manual and B2. Varian manual). 
The manual contained detailed instructions on how to perform the dose calculations and which data to return to the audit team. Each institute was asked to perform their own QA measurements of the audit plans well in advance, ensuring that the plans could be delivered at that particular institute. Prior to the audit visit, the DICOM files of the audit plans were checked by the audit team for unexpected changes in plan parameters.

\subsection{Scheduling and logistics}

To improve the efficiency of the audit, radiotherapy institutes in the same geographical region were clustered as much as possible (see NCS report 23, Figure 1 [7]). For each institute, the date of the audit was based on the global planning of the audit and the availability of the institute's linacs. Since the measurement equipment had to be returned to its owner every week and the radiochromic films had to be scanned within one week of irradiation, we attempted to schedule several audits in one week.

The audits were performed by two auditors who were scheduled based on their availability and domestic location. To guarantee independent measurements, the audit team members did not audit their own institute. The audit visits took place within one year (October 2014 to August 2015).

The measurement equipment was transported by a courier company or an audit team member. To ensure sufficient acclimatisation, the measurement equipment was delivered to the institute one or more days prior to the audit visit and was stored, for at least a number of hours, in the treatment room of the linac to be used for the audit measurements.

\subsection{Resources}

The organization, implementation and processing of such a national audit requires a minimum level of resources in terms of finance, equipment and manpower. The Dutch radiotherapy institutes were asked to pay a fee to participate to the audit. This fee was spent on the purchase of films, the calibration and transportation of the measurement equipment and the travel costs of the auditors. The phantom, ionisation chamber, 2D array and laptop were supplied without charge by one of the radiotherapy institutes. The institutes kindly provided to the members of the audit team the time required for their visits. 


\section{Materials and methods}

\subsection{Available TPS linac combinations in the Netherlands}

There is a range of linac - TPS vendor combinations in clinical use in the Netherlands (Table 3.1). All radiotherapy institutes have a $6 \mathrm{MV}$ beam available, but there is a wide range of higher energy beams available. For comparative and logistical reasons, the audit was performed for $6 \mathrm{MV}$ beams only. If the audit for this energy is successful it may safely be assumed that the QA procedure for higher energies should also comply.

Table 3.1 Available linac (6 MV) and vendor - TPS combinations in the Netherlands at the time of the audit measurements (October 2014 - August 2015).

\begin{tabular}{|llll|}
\hline Linac vendor & TPS system & $\begin{array}{l}\text { Number of } \\
\text { institutes }\end{array}$ & RTP import \\
\hline Elekta & Elekta, Monaco & 2 & Not possible $^{*}$ \\
\hline Elekta & Elekta, Oncentra & 2 & DICOM \\
\hline Elekta & Philips, Pinnacle & 10 & $\begin{array}{l}\text { Pinnacle file } \\
\text { format }\end{array}$ \\
\hline Elekta & Raysearch, & 1 & DICOM \\
& Raystation & & \\
\hline Varian & Varian, Eclipse & 5 & DICOM \\
\hline Varian & BrainLab, iPlan & 1 & Not possible \\
\hline Tomotherapy ${ }^{* *}$ & $\begin{array}{l}\text { Accuracy, } \\
\text { Tomotherapy }\end{array}$ & 1 & \\
& $\begin{array}{l}{ }^{*} \text { DICOM import is possible from version } 5.1 \\
{ }^{* *} \text { Tomotherapy was not included in this audit. }\end{array}$ & \\
\hline
\end{tabular}

\subsection{Treatment planning}

Treatment plans (IMRT and/or VMAT) of different complexity were generated for the audit: simple IMRT and VMAT (based on a simple cervical tumour), complex IMRT and VMAT (based on a head-and-neck tumour) and stereotactic VMAT (based on a brain tumour) plans. For each plan, the institute was asked if plans with similar complexity were used clinically and if the plan under consideration would have been approved to be used clinically. This information was taken into account during the analysis of the results. 
The intention of the audit was to include all available Elekta and Varian linac types in the audit. Therefore, for each plan a 'preferable' type of linac was assigned. To this end, the available linacs were grouped into two types, based on their technical advancement:

type 1: Elekta MLCi(2) or Varian Clinac; type 2: Elekta Agility or Varian TrueBeam.

Whenever possible, the simple plans were delivered on a type 1 linac, the complex and stereotactic plans on a type 2 linac. Many institutes have both type 1 and type 2 linacs available, but particular licenses (e.g. VMAT) varied.

Separate treatment plans were generated for Elekta and Varian linacs keeping the planning parameters as similar as possible (Table 3.2). For the Varian linacs, the same treatment plans (without jaw tracking) could be delivered on the type 1 (Clinac) and type 2 (TrueBeam) linacs.

Due to the differences in the design of the Elekta linac head, different plans had to be generated for the type $1(\mathrm{MLCi}(2))$ and the type 2 (Agility) Elekta linacs.

Due to the lack of plan import options, plans had to be generated in Monaco and iPlan by the institute (3 out of 22 institute sites), hampering a 100\% one-to-one comparison with the audit plans. However, these plans were based on the same patient CT data sets as the audit plans.

\subsubsection{General planning parameters}

The audit treatment plans were generated in either Pinnacle (Philips Medical Systems International B.V., Best, the Netherlands) (for the Elekta linacs) or Eclipse (Varian Medical Systems, Palo Alto, California) (for the Varian linacs). This choice was based on the frequency of these combinations within the Netherlands (Table 3.1).

The plan characteristics (e.g. beam angle, MU) of the different plans are listed in Table 3.2. The IMRT plans used either a step-and-shoot (Elekta) or dynamic leaf (Varian) technique. For the stereotactic plan, the high dose area was at least $3 \mathrm{~cm}$ in diameter, which was large enough for accurate measurement

The classification of the plans (simple, complex and stereotactic) was ensured using different tumour sites in the preparation phase. To assess the complexity of the plans, e.g. the segment shape, leaf motion and dose distribution of the plans were evaluated visually. To ensure accurate dose measurements in the audit phantom, the planned dose at the centre of the phantom was made as homogeneous as possible, avoiding steep dose gradients, and the isocentre was located in the high dose volume. 
Table 3.2 Plan characteristics.

\begin{tabular}{|c|c|c|c|c|c|}
\hline Parameter & Simple & & Complex & & $\begin{array}{l}\text { Stereo- } \\
\text { tactic }\end{array}$ \\
\hline Technique & $\begin{array}{l}\text { IMRT, } \\
5 \text { beams }\end{array}$ & $\begin{array}{l}\text { VMAT, } \\
1 \text { arc }\end{array}$ & $\begin{array}{l}\text { IMRT, } \\
7 \text { beams }\end{array}$ & $\begin{array}{l}\text { VMAT, } \\
2 \text { arcs }\end{array}$ & $\begin{array}{l}\text { VMAT, } \\
1 \text { arc }\end{array}$ \\
\hline $\begin{array}{l}\text { Preferable linac } \\
\text { Elekta } \\
\text { Varian }\end{array}$ & $\begin{array}{l}\text { type 1: } \\
\text { MLCi(2)/ } \\
\text { Clinac }\end{array}$ & $\begin{array}{l}\text { type 1: } \\
\text { MLCi(2)/ } \\
\text { Clinac }\end{array}$ & $\begin{array}{l}\text { type 2: } \\
\text { Agility/ } \\
\text { TrueBeam }\end{array}$ & $\begin{array}{l}\text { type 2: } \\
\text { Agility/ } \\
\text { TrueBeam }\end{array}$ & $\begin{array}{l}\text { type 2: } \\
\text { Agility/ } \\
\text { TrueBeam }\end{array}$ \\
\hline Energy (MV) & 6 & 6 & 6 & 6 & 6 \\
\hline Gantry angles $\left({ }^{\circ}\right)$ & $\begin{array}{l}-144,-72 \\
0,72,144\end{array}$ & $178-182$ & $\begin{array}{l}-150,-110 \\
-50,0,50 \\
110,150\end{array}$ & $\begin{array}{l}178-182 \\
182-178\end{array}$ & $178-182$ \\
\hline Collimator angle $\left({ }^{\circ}\right)$ & 20 & 20 & 20 & 20 & 20 \\
\hline $\begin{array}{l}\text { Dose (cGy) at isoc } \\
\text { Elekta plans } \\
\text { Varian plans }\end{array}$ & $\begin{array}{l}184.5 / \\
188.5\end{array}$ & $\begin{array}{l}193.2 / \\
181.5\end{array}$ & $\begin{array}{l}144.8 / \\
146.5\end{array}$ & $\begin{array}{l}146.3 / \\
143.6\end{array}$ & $\begin{array}{l}353.8 / \\
357.5\end{array}$ \\
\hline $\begin{array}{l}\text { Total \# MU * } \\
\text { Elekta plans } \\
\text { Varian plans }\end{array}$ & $\begin{array}{l}418.6 / \\
878.3\end{array}$ & $\begin{array}{l}391.4 / \\
565.0\end{array}$ & $\begin{array}{l}511.7 / \\
1313.1\end{array}$ & $\begin{array}{l}388.8 / \\
461.8\end{array}$ & $\begin{array}{l}675.0 / \\
710.5\end{array}$ \\
\hline $\begin{array}{l}\text { CPs / segments ** } \\
\text { Elekta plans } \\
\text { Varian plans }\end{array}$ & $\begin{array}{l}35 \\
643\end{array}$ & $\begin{array}{l}90 \\
178\end{array}$ & $\begin{array}{l}60 \\
1632\end{array}$ & $\begin{array}{l}180 \\
356\end{array}$ & $\begin{array}{l}90 \\
178\end{array}$ \\
\hline $\begin{array}{l}\text { *1 MU = } 1 \text { cGy @ SSD } \\
\text { ** Number of segments } \\
\text { IMRT and VMAT (Elekte }\end{array}$ & $\begin{array}{l}\text { cm @ dm } \\
\text { ep and Shc } \\
\text { ian) }\end{array}$ & lekta) IM & mber of co & nts for dyn & If (Varian) \\
\hline
\end{tabular}

\subsection{Audit preparation}

\subsubsection{Distribution of audit treatment plans}

The audit treatment plans, the CT scan and structure data set were distributed to the institutes. 
The Elekta treatment plans were distributed in either Pinnacle file format (to Pinnacle TPS) or in DICOM format (to Raystation, RaySearch, Stockholm, Sweden and Oncentra, Elekta, Stockholm, Sweden). The Varian treatment plans were distributed in DICOM format (Eclipse). Fields with sizes of $10 \times 10 \mathrm{~cm}^{2}$ (calibration) and $25 \times 25 \mathrm{~cm}^{2}$ (pre-irradiation of array) were also provided to the institutes.

\subsubsection{Dose calculation in each institute}

After importing the CT and structure data sets of the audit phantom and the audit treatment plans into the institute TPS system, the dose was calculated on a dose grid of $2 \times 2 \times 2 \mathrm{~mm}^{3}$. The density of the phantom CT was overridden with relative electron density $=1.016 \mathrm{~g} / \mathrm{cm}^{3}$ (or mass density $=1.04 \mathrm{~g} / \mathrm{cm}^{3}$ ) according to the phantom manual [8]. All other calculation settings (e.g. dose algorithm, correction for treatment table) followed the clinically applied protocol of the institute. The computed dose distribution was returned to the audit team in order to be used for the analysis of the audit results. The definition of monitor units (MU) used for the audit plans was in accordance with the nationally recommended standard, i.e. 1 $M U=1 \mathrm{cGy}$ at $d_{\max }$ for a $10 \times 10 \mathrm{~cm}^{2}$ field at $S S D=100 \mathrm{~cm}$. In institutes where the $M U$ definition differed significantly from the national definition, the MUs were adjusted by a scaling factor to maintain a comparable delivered target dose level. This is especially important for dynamic plans, given the co-dependence between the delivered dose and the dynamic behaviour of the linac.

\subsubsection{Institute QA of treatment plans}

Prior to the audit visit, each institute performed QA measurements of the audit plans according to their local protocol and equipment (Table 3.3).

To be able to compare the institute QA with the results of the audit, the audit team requested to perform an additional global gamma analysis for each plan using the same criteria as applied for the audit measurements (section 3.5, Appendix 1B). 
Table 3.3 QA measurement devices used by the institutes in preparation of the audit visit.

\begin{tabular}{|l|ll|}
\hline QA equipment & Vendor & Number of institutes \\
\hline ArcCHECK & Sun Nuclear Corporation & 2 \\
\hline Delta4 & Scandidos AB & 5 \\
\hline EPIDdosimetry & Elekta & 1 \\
\hline EPIDdosimetry & Varian & $2^{*}$ \\
\hline $\begin{array}{l}\text { Film (EBT3) and point } \\
\text { dosimetry }\end{array}$ & PEO/PTW & 5 \\
\hline MatriXX & IBA & $4^{* *}$ \\
\hline Octavius-2D & PTW & 2 \\
\hline Octavius-4D & PTW & \multicolumn{2}{l|}{$\begin{array}{l}\text { * Film and ionisation chamber are used in combination with a slab phantom or the Octavius phantom. } \\
\text { ** In one institute, the PTW 729 array is used in combination with a slab phantom. }\end{array}$} \\
\hline
\end{tabular}

\subsection{Audit equipment and measurements methods}

The audit measurements were performed according to the audit measurement protocol (Appendix 1A).

\subsubsection{Phantom}

All measurements were performed in the Octavius phantom (Octavius ${ }^{\circledR}$ II, PTW Freiburg $\mathrm{GmbH}$, Freiburg, Germany). This phantom was chosen as it is robust, suitable for array, point and film measurements, easily transportable and straight forward to calibrate and use [9].

The phantom consists of separate pieces: one upper and two lower sections. One lower section is dedicated to array measurements and the other to point- and film measurements. The point- and film lower section is solid, while the array section has an air cavity to compensate for the reduced sensitivity of the array when irradiated from below. The relative electron density of this phantom is $1.016 \mathrm{~g} / \mathrm{cm}^{3}$ and, the mass density is $1.04 \mathrm{~g} / \mathrm{cm}^{3}[8,10]$. 

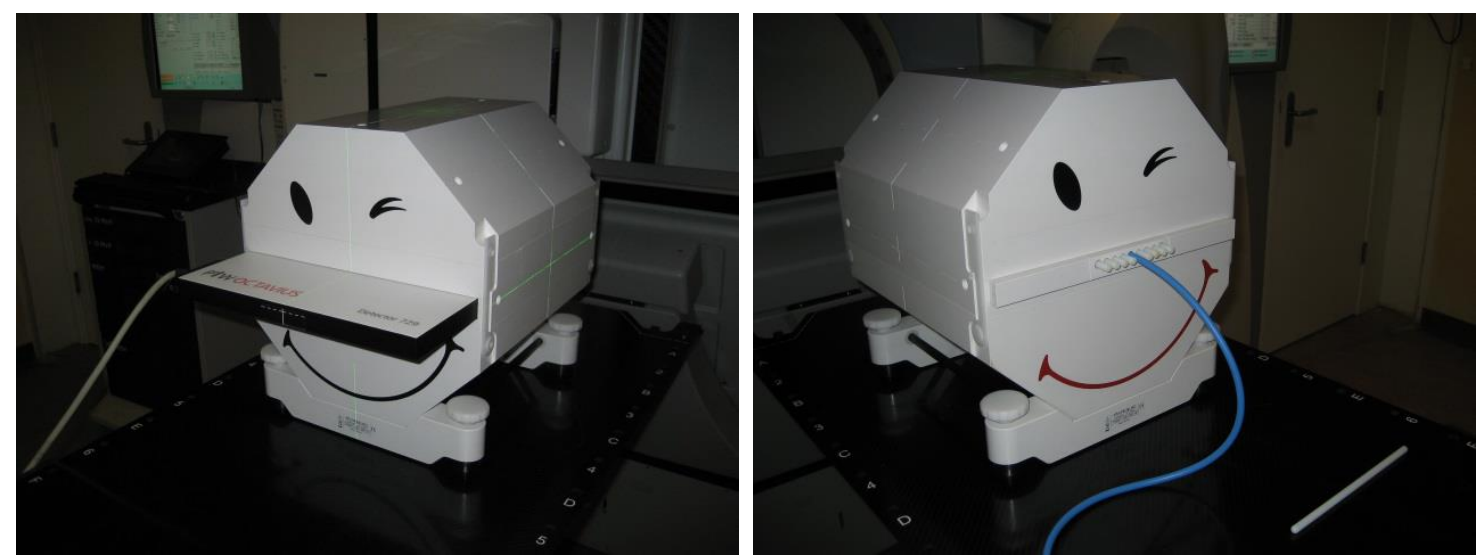

Figure 3.1 The Octavius phantom with the array lower half section (left) and with the ionisation chamber insert and film lower half section (right).

\subsubsection{Ionisation chamber measurement}

\section{Measurement procedure}

The point dose was measured using a $0.016 \mathrm{~cm}^{3}$ PinPoint ionisation chamber (TN31016 PTW Freiburg GmbH, Freiburg, Germany) in combination with an electrometer (Unidos ${ }^{\text {Webline, }}$ PTW Freiburg GmbH, Freiburg, Germany). The ionisation chamber was placed in the phantom, using the corresponding lower section of the phantom and the ionisation chamber insert. Hence, the chamber was aligned with the linac isocentre using the in-room lasers, being placed at the centre of the phantom.

Before each measurement session, the chamber was pre-irradiated with approximately 8 Gy before the background correction of the electrometer was performed. This operation consisted in measuring for 30 seconds without irradiation, which should result in a reading of less than $0.1 \mathrm{pC}$.

Next, the reference field of $10 \times 10 \mathrm{~cm}^{2}$ (200 MU) was delivered twice and the reading was recorded. Finally, the audit treatment plans were delivered and the reading of the complete plan was recorded. Afterwards, the reference field of $10 \times 10 \mathrm{~cm}^{2}$ (200 MU) was again delivered twice to check for any variation in machine output.

\section{Formalism}

The readings were converted to absolute dose according to the $k_{Q}$ formalism [11]: 


$$
D_{W}=M_{c o r} N_{D_{w}, o_{60}} k_{Q}
$$

The volume recombination correction for the used ionisation chamber (PinPoint), needed to determine $M_{\text {cor }}$, was determined for a $10 \times 10 \mathrm{~cm}^{2}$ reference field using the two voltage method [11] in an Elekta Precise linac. The measured value was 1.002.

The procedure for the point dose measurement deviates from the NCS-18 protocol in two aspects:

- the used ionisation chamber is not part of NCS-18, therefore the generic $k_{\mathrm{Q}}$ fit of NCS-18 is not applicable;

- the audit treatment plan point doses are measured in non-standard fields, which are considerably different from the $10 \times 10 \mathrm{~cm}^{2}$ reference field. This might affect the response of the ionisation chamber for these fields.

The first deviation was accounted for by directly measuring the $k_{\mathrm{Q}}$ factor, specifically for the PinPoint ionisation chamber used in the audit measurements. The $k_{\mathrm{Q}}$ factor is determined using the ratio of two cross-calibrations against a waterproof Farmer chamber (TN 30013, PTW Freiburg GmbH, Freiburg, Germany); one in a $10 \times 10 \mathrm{~cm}^{2} 6 \mathrm{MV}$ linac reference field (Elekta Precise) and one in a $10 \times 10 \mathrm{~cm}^{2}{ }^{60} \mathrm{Co}$ reference field. The measured and employed value of $k_{Q}$ was 0.998 .

For the second deviation from NCS-18, the method proposed by Alfonso et al. [12] was used to assess the differences in ionisation chamber response between the radiation fields of the audit treatment plans and reference fields. For this purpose, the ratio of the corrected readings for two detectors (the PinPoint ionisation chamber and a diamond detector (TN 60019, PTW Freiburg GmbH, Freiburg, Germany) at the point of measurement in a cubic water phantom was determined for several audit treatment plans and for the $10 \times 10 \mathrm{~cm}^{2}$ reference field. The difference between a particular measured ratio for the respective audit plans and the reference field ratio, is an estimate of the 'non-standard field effect' change in response of the ionisation chamber response for the relevant audit plan. The maximum estimated non-standard field effect was $0.9 \%$, which is much smaller than differences, of up to $1.9 \%$, reported previously $[13,14]$. This effect was taken into account in the uncertainty budget (Appendix 1C, Table C. 1). The total uncertainty of the point measurement is $2.1 \%$ (2 standard deviations, see Appendix 1C, Table C. 1), which justifies the use of a $\pm 3.0 \%$ tolerance level for the point measurements. 
The relative difference, $\Delta$, between the absorbed dose, as measured in the isocentre by the ionisation chamber, and the planned dose, as provided by the institute, was calculated. This value was multiplied by the ratio of the calculated dose and the measured dose for the $10 \times 10 \mathrm{~cm}^{2}$ field, to determine the reported $\Delta_{\mathrm{N}}$.

\subsubsection{Array measurement}

\section{Measurement procedure}

A 2D-array (Octavius ${ }^{\circledR}$ || 729, PTW Freiburg GmbH, Freiburg, Germany) with 729 planeparallel ionisation chambers was used for the array measurements. The array measurements were recorded by the VeriSoft software $\left(\right.$ VeriSoft $^{\circledR}$, version 6.1, PTW Freiburg GmbH, Freiburg, Germany).

The 2D-array was placed in the Octavius phantom with the corresponding lower section. The array was pre-irradiated with a field of $25 \times 25 \mathrm{~cm}^{2}$ (800 MU), after which a zero background correction measurement was performed. The reference field of $10 \times 10 \mathrm{~cm}^{2}$ (200 MU) was then delivered. Next, the plans were delivered and each plan was recorded in its entirety; separate beam or arc data was not recorded. Afterwards, the reference field of $10 \times 10 \mathrm{~cm}^{2}$ was delivered and recorded again to check for machine output variations.

3D global gamma analysis

The analysis of the measurements was performed in VeriSoft, a software package for comparing two dose planes which includes a gamma index evaluation algorithm.

The 2D audit measurements from the array and film (see section 3.4.4) were compared to the corresponding 2D dose plane derived from the $3 \mathrm{D}$ dose distribution calculated by the institute TPS by means of a 3D global gamma analysis. Verisoft allows the user to adjust various settings, including the dose and distance to agreement acceptance criteria as well as a user-defined scaling factor ( $k_{\text {user }}$, see below).

For the simple and complex IMRT and VMAT plans, the acceptance criteria were chosen to be $3 \mathrm{~mm}$ and 3\%, as recommended by the NCS report 24 [4]. For the stereotactic plan, the criteria were $1 \mathrm{~mm}$ and $5 \%$, because for this type of treatment the geometrical accuracy is more important than the dosimetric accuracy. 
A plan passed the gamma test if more than $95 \%$ of the evaluated measurement points met the gamma criteria. Dose regions below a threshold of $10 \%$ of the maximum measured dose were not included in the analysis.

The factor $k_{\text {user }}$ was defined as the ratio of the calculated isocentre dose and the average of dose measured by the central ionization chamber of the 2D-array, before and after measuring each plan, for a $10 \times 10 \mathrm{~cm}^{2}$ field. By using $\mathrm{k}_{\text {user }}$ the $2 \mathrm{D}$ measurement is normalized to the corresponding dose plan derived from the $3 D$ computed dose distribution ruling out daily variations of the linac output.

Prior to gamma comparison, the measured and calculated dose planes were geometrically aligned by an automatic registration optimization procedure in Verisoft. If the translation between measured and calculated dose planes was larger than $1 \mathrm{~mm}$, the shift was applied to the calculated dose plane. For 19 of the total 90 measurements a translation larger than 1 $\mathrm{mm}$ was found (absolute average of $1.3 \pm 0.2 \mathrm{~mm}$ and $1.5 \pm 0.2 \mathrm{~mm}$ in the two plane directions respectively) and explained by the stand of the lasers in the linac bunker used to align the phantom.

\subsubsection{Film measurement}

\section{Measurement procedure}

Film measurements were performed using radiochromic films (Gafchromic EBT3, Ashland Specialty Group, Wayne USA) from a single batch (Lot\#07291401). The films were placed in the Octavius phantom film insert. The alignment of the film with the isocentre was guaranteed by puncturing holes through the top of the insert. Next, the film insert, with the film included, was placed in the Octavius phantom with the corresponding lower section. Each plan was delivered in its entirety to a single film.

At each institute, calibration films were irradiated at one of the linacs used for the audit. Quarters of a film were irradiated with a $10 \times 10 \mathrm{~cm}^{2}$ field for $0,200,400$ and $600 \mathrm{MU}$, respectively.

\section{Dose conversion and gamma analysis}

Films were scanned with an Epson 10000 XL (Epson Seiko Corporation, Nagano, Japan) [15]. All data modification options from the scan software (Silverfast, LaserSoft Imaging Inc., Sarasota FL, USA) were turned off. The spatial resolution was set to $72 \mathrm{dpi}(0.35 \mathrm{~mm})$, the optical resolution was 16 bit for each color channel. For this audit, the film orientation was in 
landscape mode [16-20]. The films of each institute were scanned within a 30-minute time frame, starting with 8 warm-up scans of the empty glass plate. The time between irradiation and scanning was at least one week, which minimizes post- exposure growth effects [21-23]. The optical density (OD) per pixel was calculated as the $\log _{10}$ ratio of the intensities of the empty glass plate (warm-up) scan and the film scan. In this way, the optical density was corrected for imperfections in the glass plate and the scanner optical system. The lateral scan effect was corrected by using a look-up table, according to literature [15,24].

The TPS dose of the $10 \times 10 \mathrm{~cm}^{2}$ field calculated by the institute and the calibration films were used to create a unique calibration curve for each institute. Film calibration curves were produced in dedicated film analysis software, FilmQA Pro (Ashland, Covington, KY, USA). A calibration function for all three color channels was used [25].

After processing all corrections, irradiated films were converted to dose-data according to the institute-specific calibration curve (FilmQAPro) [25]. The 2D dose plane from the red channel was compared to the corresponding dose plane derived from the $3 \mathrm{D}$ dose cube calculated by the institute's TPS using the gamma evaluation tool present in VeriSoft (see section 3.4.3). The gamma analysis was performed for the same gamma criteria as for the array.

In contrast to the array measurement the automatic alignment between film and measurement was always applied because the marks on the film used to define its centre could not always be accurate determined in Verisoft (absolute average of $0.5 \pm 0.4 \mathrm{~mm}$ and $0.6 \pm 0.5 \mathrm{~mm}$ in the two plane directions respectively).

\subsection{Analysis of results}

The pass or fail agreement between each audit results (per measurement method) and the institute QA results were evaluated per dosimeter and plan type. Moreover, the audit results of the measurements performed on Elekta en Varian linacs were separately analysed because the plans delivered on these machines were generated separately.

The median values of all audit measurement results, including the median of the mean gamma value and the pass rate, was calculated per measurement method and per plan type. As described above, the dose plane derived from the $3 \mathrm{D}$ dose cube calculated in the institute's treatment planning system was used for the gamma analysis.

Less than three measurements per plan type was considered statistically insufficient, therefore to have at least three measurement results per plan type, the results for the Varian 
Clinac and TrueBeam linacs were combined. This was allowed because the same plans were delivered on both linacs.

Three institutes did not deliver treatment techniques similar to that of the simple and complex IMRT plans. Techniques similar to the simple VMAT plan could not be found in two institutes, and one institute did not deliver plans similar to the complex VMAT plan. Nevertheless, the measurement results of these plans are included in the analysis.

Due to the fact that the DICOM import in the treatment planning system was not always possible, three institutes used self-created plans. The results of these audit measurements were not included in the group analysis. 


\section{Results}

In total 82 plans were measured with each measurement method for 22 radiotherapy locations: simple IMRT: 18; simple VMAT: 14; complex IMRT: 18; complex VMAT: 16, stereotactic VMAT: 16 times.

\subsection{Analysis per plan type and dosimeter}

\subsubsection{Pass/Fail agreement between audit and institute QA}

Figure 4.1 shows the agreement between results of the audit (three measurement methods) and the institute QA.
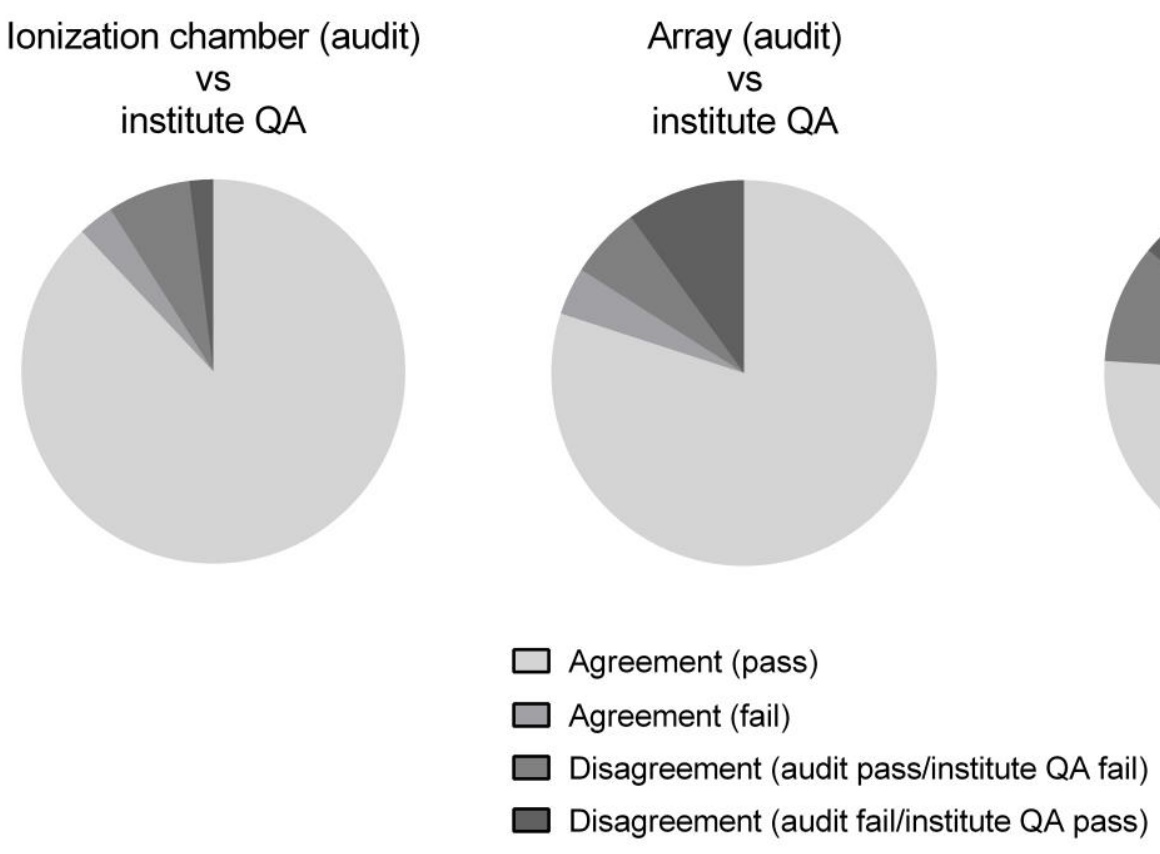

Figure 4.1 Pie-charts showing the agreement between results of the audit and the institute QA for all plans. The acceptance criteria were: a gamma pass rate $\geq 95 \%(5 \%, 1 \mathrm{~mm}$ for the stereotactic plan and $3 \%, 3 \mathrm{~mm}$ for the other plans) for the 2D measurements (array and film); a relative difference within $\pm 3 \%$ for the point measurements (ionisation chamber).

The majority of the measurements included in the analysis was in agreement with the institute QA results (ionization chamber: 74/82; array: 69/82; film: 62/82). The institute QA 
results agreed with the audit and were within the acceptance criteria (Agreement (pass)) for 72 (ionization chamber), 66 (array) and 62 (film) measurements, while 2 (ionization chamber), 3 (array) and 0 (film) of the 82 cases agreed but failed to pass the acceptance criteria (Agreement (fail)).

A disagreement between the audit and institute QA results was found for 8 (ionization chamber), 13 (array) and 20 (film) cases. Of these, 6 (ionization chamber), 5 (array) and 8 (film) failed the audit measurement while each of the institute $Q A$ plans passed (Disagreement (audit fail/ institute QA pass)). Vice versa, 2 (ionization chamber), 8 (array) and 12 (film) plans passed the audit and failed the institute QA (Disagreement (audit pass/ institute QA fail)).

The proportion of plans that both passed the audit and failed the institute QA result is consistent among the three dosimeters; however, where the audit failed and the institute QA passed, a larger variation is observed.

Figure 4.2 shows the results of the audit and the institute QA per plan type and per measurement method. Overall, the most "Agreements (pass)" were found for the stereotactic VMAT plan: only at one institute this plan failed the institute QA result. "Agreement (fail)" results were found for the simple VMAT (0 (ionization chamber); 1 (array); 0 (film)) and complex IMRT (1 (ionization chamber); 1 (array); 0 (film)) and VMAT (1 (ionization chamber); 1 (array); 8 (film)) plans. However, some of these plan types were marked as "not used clinically" by certain institutes.

The number of disagreements (either audit pass/institute QA fail or audit fail/institute QA pass) are depending on plan type and measurement method. Most disagreements occurred in the film results (up to $33 \%$ for the complex IMRT plan). Less disagreements occurred in the evaluations of the array (up to $29 \%$ for the simple VMAT plan) and ionization chamber (up to $11 \%$ for the simple IMRT plan) measurements.

When considering the array results, a larger number of measurements failed for the simple VMAT plan compared to the other plan types. For this plan, the reason for failing can be explained by the directional dependence of the array response [26-28]: a substantial number of MU's was delivered at 90 and 270 degrees, the angles of decreased detector response. 
Ionization chamber (audit)

XS

Institute QA

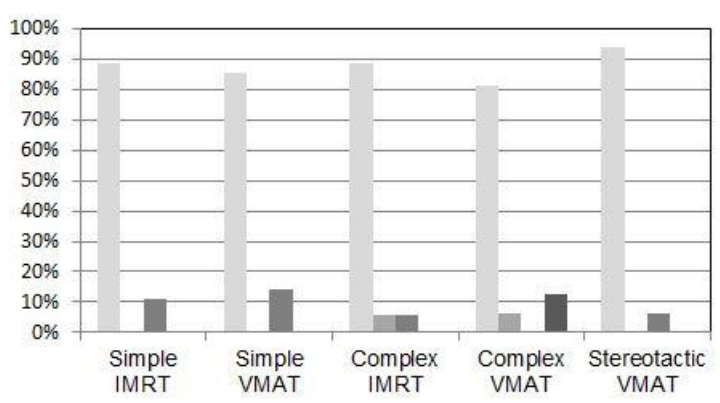

Film (audit)

Institute QA

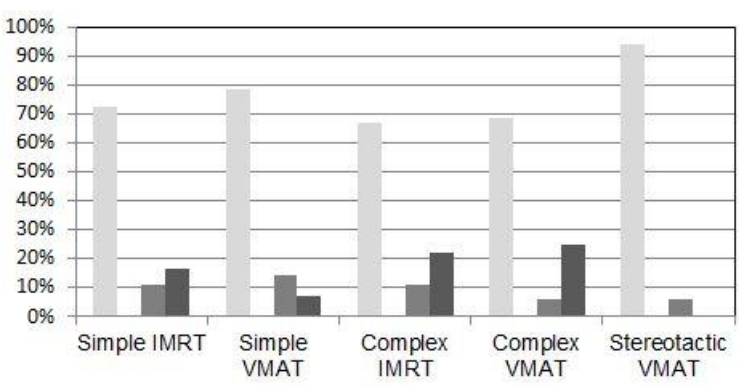

Array (audit)

VS
Institute QA

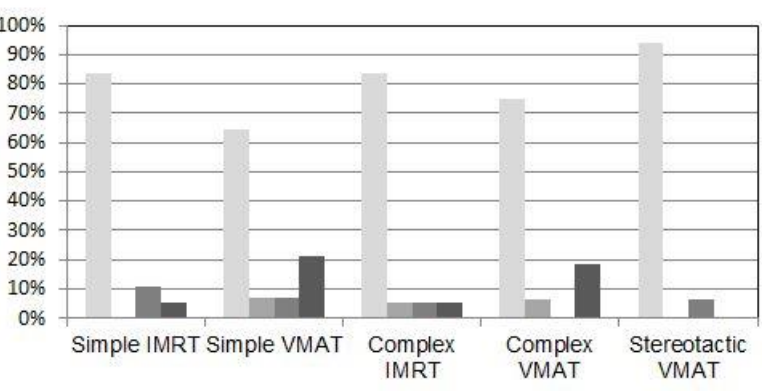

Agreement (pass)

Agreement (fail)

Disagreement (audit pass/ institute QA fail)

Disagreement (au dit fail / institute QA pass)

Figure 4.2 Results of the audit and institute QA grouped by plan type for each measurement method. The percentage was calculated with respect to the total amount of measured plans per plan type (simple IMRT: 18; simple VMAT: 14; complex IMRT: 18; complex VMAT: 16, stereotactic VMAT: 16). The acceptance criteria were: a gamma pass rate $\geq 95 \%(5 \%, 1 \mathrm{~mm}$ for the stereotactic plan and $3 \%, 3 \mathrm{~mm}$ for the other plans) for the $2 \mathrm{D}$ measurements (array and film); a relative difference within $\pm 3 \%$ for the point measurements (ionisation chamber).

\subsubsection{Distribution of the audit and institute $Q A$ results}

Scatter plots indicating the median and interquartile range of the audit and institute QA results are shown in Figure 4.3 and Table 4.1. The median of the relative differences between calculation and the ionisation chamber measurements $\left(\Delta_{N}\right)$ was $(-1.3 \pm 0.6)$ (average of the median value of each plan type). The median of the gamma pass rates was close to $100 \%$, for both the film $(99.1 \pm 0.6)$ and array $(99.7 \pm 0.4)$ measurements as well as the institute $Q A$ result $(99.7 \pm 0.3)$. 
For the array measurements, the range of the pass rates observed for the simple $(75.8 \%$ $100 \%)$ and complex (83.3\% - $100 \%)$ VMAT plans was larger than the institute QA result range (simple VMAT: 80.3\% -100\%; complex VMAT: 93.2\%-100\%) (Figure 4.3).

For the film measurements, a larger number of outliers were found than for the other audit measurement methods (Figure 4.3). Furthermore, the pass rates of several outliers were extremely low $(<50 \%)$. For this reason, the results of the audit film measurements were considered not reliable (see section 5.3) and therefore not reported in the analysis per linac type.

The audit results for the three institutes that used self-created plans, excluded from the overall analysis, were within the ranges reported in Table 4.1.

The audit results of the plans that were marked as "not used clinically" by some institutes were within the $25 \%-75 \%$ percentile of the pass rates, except for one measurement of the complex IMRT plan.
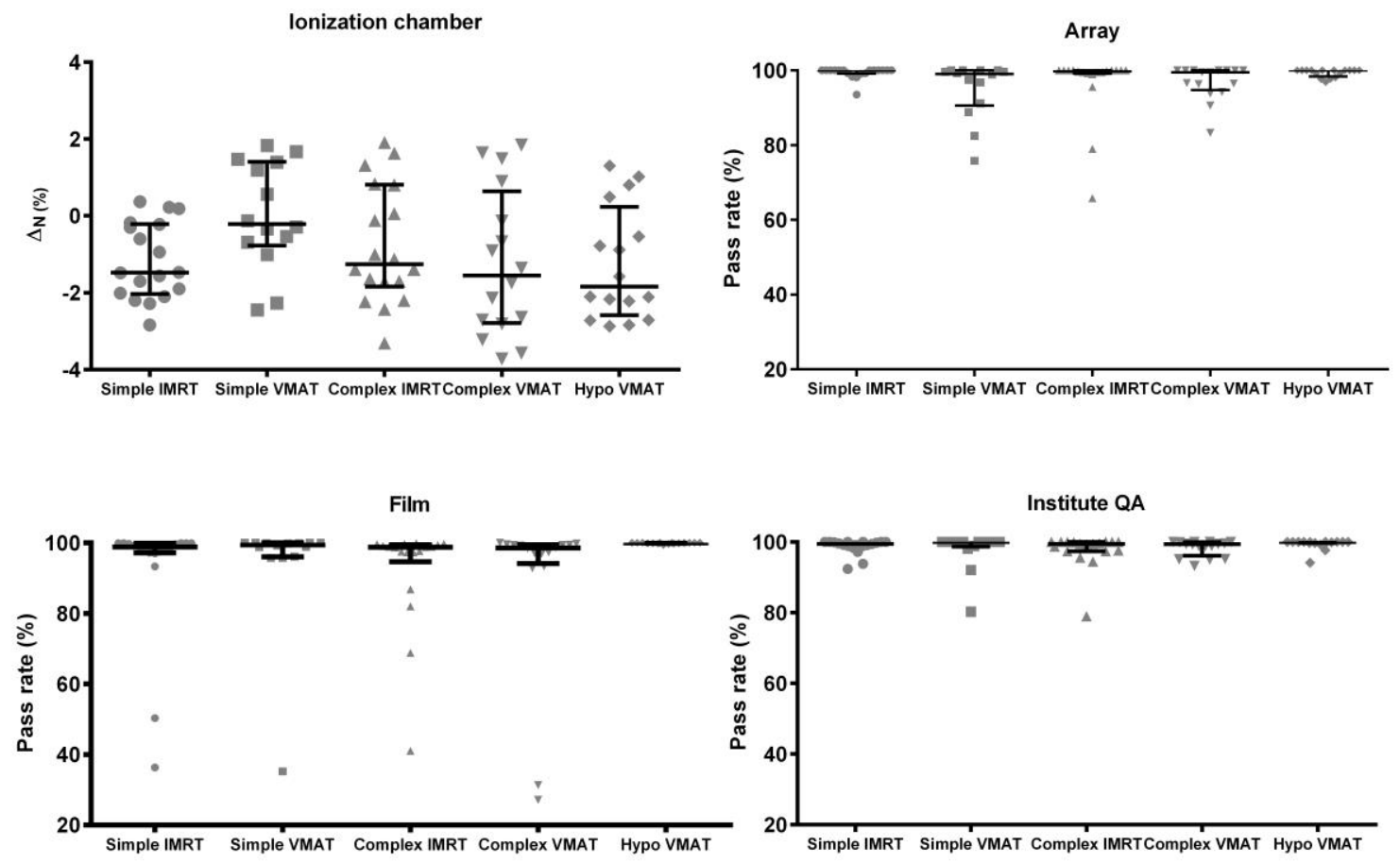

Figure 4.3 Scatter plot indicating the median and interquartile range of the audit results for all plans for the ionization chamber (upper left), array (upper right), film (lower left), and institute QA result (lower right) measurements. 
Table 4.1 Median values of the audit and institute QA results for all plans.

\begin{tabular}{|c|c|c|c|c|c|}
\hline Plan type & $\begin{array}{c}\text { Number of } \\
\text { measure- } \\
\text { ments }\end{array}$ & $\begin{array}{l}\text { Ionization } \\
\text { chamber }\end{array}$ & Array & Film & Institute QA \\
\hline & & $\Delta_{N}(\%)$ & & Pass rate (\%) & \\
\hline & & $\begin{array}{c}\text { Median } \\
(\min -\max )\end{array}$ & $\begin{array}{c}\text { Median } \\
(\min -\max )\end{array}$ & $\begin{array}{c}\text { Median } \\
(\min -\max )\end{array}$ & $\begin{array}{c}\text { Median } \\
(\min -\max )\end{array}$ \\
\hline $\begin{array}{l}\text { Simple } \\
\text { IMRT }\end{array}$ & 18 & $\begin{array}{c}-1.5 \\
(-2.8-+0.4)\end{array}$ & $\begin{array}{c}100.0 \\
(93.5-100.0)\end{array}$ & $\begin{array}{c}98.8 \\
(36.3-99.9)\end{array}$ & $\begin{array}{c}99.5 \\
(92.4-100.0)\end{array}$ \\
\hline $\begin{array}{l}\text { Simple } \\
\text { VMAT }\end{array}$ & 14 & $\begin{array}{c}-0.2 \\
(-2.5-+1.8)\end{array}$ & $\begin{array}{c}99.1 \\
(75.8-100.0)\end{array}$ & $\begin{array}{c}99.5 \\
(35.2-100.0)\end{array}$ & $\begin{array}{c}100.0 \\
(80.3-100.0)\end{array}$ \\
\hline $\begin{array}{l}\text { Complex } \\
\text { IMRT }\end{array}$ & 18 & $\begin{array}{c}-1.3 \\
(-3.3-+1.9)\end{array}$ & $\begin{array}{c}99.8 \\
(65.8-100.0)\end{array}$ & $\begin{array}{c}98.8 \\
(41.0-99.9)\end{array}$ & $\begin{array}{c}99.5 \\
(79.0-100.0)\end{array}$ \\
\hline $\begin{array}{l}\text { Complex } \\
\text { VMAT }\end{array}$ & 16 & $\begin{array}{c}-1.6 \\
(-3.7-+1.8)\end{array}$ & $\begin{array}{c}99.5 \\
(83.3-100.0)\end{array}$ & $\begin{array}{c}98.6 \\
(27.1-100.0)\end{array}$ & $\begin{array}{c}99.4 \\
(93.2-100.0)\end{array}$ \\
\hline $\begin{array}{l}\text { Stereotactic } \\
\text { VMAT }\end{array}$ & 16 & $\begin{array}{c}-1.8 \\
(-2.9-+1.3)\end{array}$ & $\begin{array}{c}100.0 \\
(96.9-100.0)\end{array}$ & $\begin{array}{c}100.0 \\
(99.4-100.0)\end{array}$ & $\begin{array}{c}100.0 \\
(94.1-100.0)\end{array}$ \\
\hline
\end{tabular}

Table 4.2 shows per plan type the median values of the mean gamma values for the audit array and film measurements. The average of this value $(0.3 \pm 0.1)$ was quite low. For the film measurements, however, a greater number of outliers were observed than for the array results.

Table 4.2 Median values of the mean gamma value for the audit array and film measurements for all plans.

\begin{tabular}{|c|c|c|}
\hline Plan type & Array & Film \\
\hline & \multicolumn{2}{|c|}{ Mean gamma value } \\
\hline & Median $(\min -\max )$ & Median $(\min -\max )$ \\
\hline Simple IMRT & $0.3(0.2-0.5)$ & $0.4(0.3-1.3)$ \\
\hline Simple VMAT & $0.4(0.2-0.8)$ & $0.3(0.2-1.3)$ \\
\hline Complex IMRT & $0.3(0.2-0.8)$ & $0.3(0.2-1.2)$ \\
\hline Complex IMRT & $0.4(0.2-0.7)$ & $0.3(0.3-1.5)$ \\
\hline Complex VMAT & $0.2(0.1-0.4)$ & $0.2(0.1-0.4)$ \\
\hline Stereotactic VMAT & $0.3(0.2-0.5)$ & $0.4(0.3-1.3)$ \\
\hline
\end{tabular}




\subsection{Analysis per linac type - Elekta}

The total number of plans measured on Elekta linacs was 52: 23 on type 1 (MLCi) and 29 on type 2 (Agility).

\subsubsection{Pass/fail agreement between audit and institute QA results}

In Figure 4.4 the results of the audit and the institute QA for the measured plans is presented.

For the array and ionisation chamber 42 and 47 measurements respectively agreed and passed ("Agreement (pass)"), while respectively 2 and 1 of the 52 cases agreed and failed ("Agreement (fail)").

A disagreement was found in 8 (array) and 4 (ionization chamber) of measurements, where a pass was found in either the audit measurements or the institute QA result.

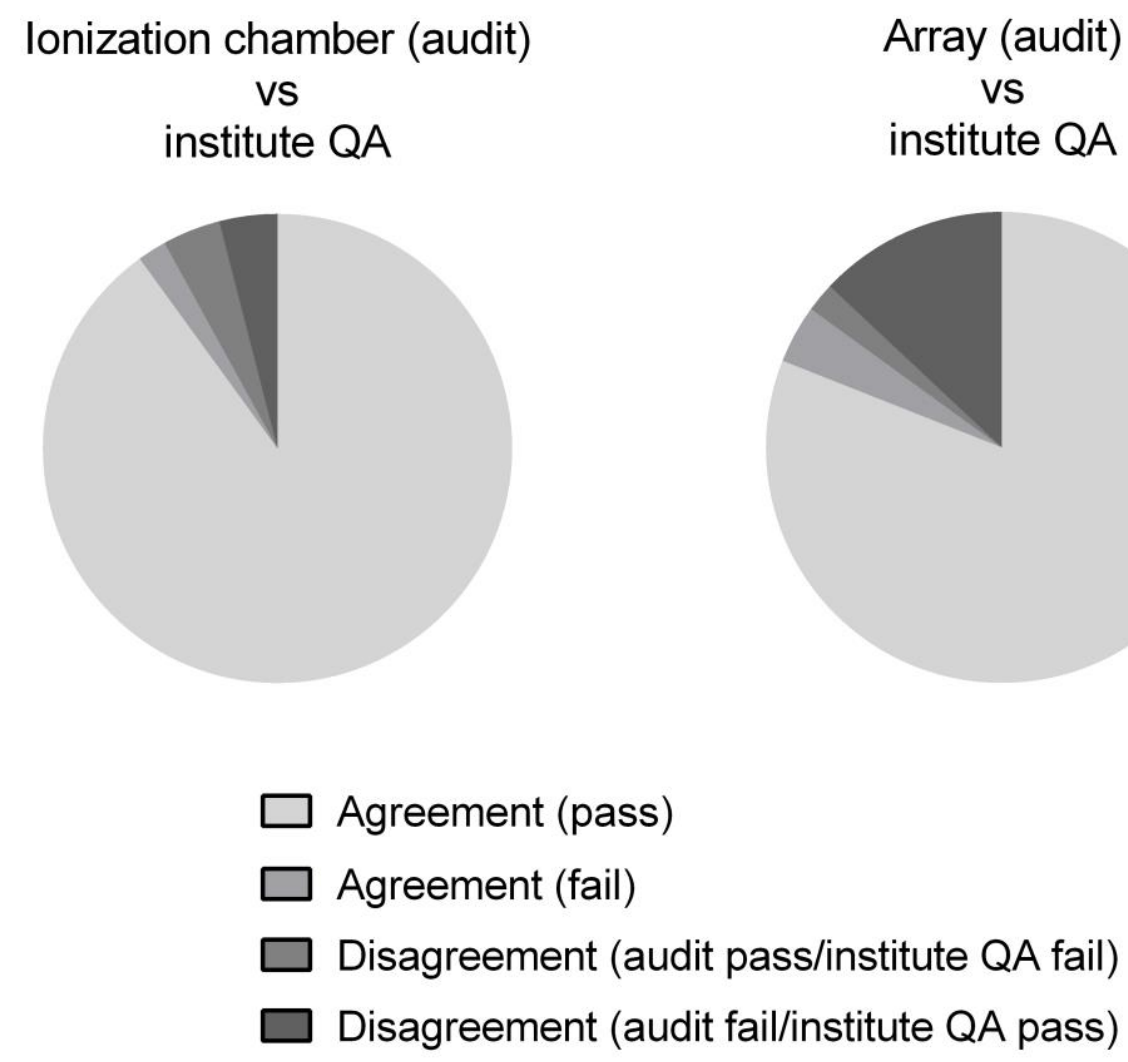

Figure 4.4 Pie-charts showing the results of the agreement between the audit and institute QA measurements, for point and array measurements for all Elekta plans. The acceptance criteria were: a gamma pass rate $\geq 95 \%(5 \%, 1 \mathrm{~mm}$ for the stereotactic plan and $3 \%, 3 \mathrm{~mm}$ for the other plans) for the array measurements; and a relative difference $\pm 3 \%$ for the ionisation chamber measurements. 


\subsubsection{Distribution of the audit and institute QA results}

The results of the measurements performed with the Elekta linacs per plan type are summarized in Table 4.3.

The median relative difference of the ionisation chamber measurements $\left(\Delta_{N}\right)$ was $-1 \%$ and $-2 \%$ on type 1 and type 2 linacs respectively. Overall the median pass rates per plan type were well above $95 \%$ for the array measurement. The results of the simple VMAT plan, however, showed more evaluations with a low pass rate (also mentioned in paragraph 4.1.1). This larger range was not observed in the institute QA results, except for one outlier.

For the Agility linacs, a large range of the results was observed for the complex VMAT plan, which was also observed in the institute QA.

Table 4.3 Median values of the audit and institute QA results for the Elekta plans (MLCi(2) or Agility).

\begin{tabular}{|c|c|c|c|c|c|}
\hline Plan type & $\begin{array}{l}\text { Linac } \\
\text { type }\end{array}$ & $\begin{array}{c}\text { Number of } \\
\text { measure- } \\
\text { ments }\end{array}$ & $\begin{array}{l}\text { Ionization } \\
\text { chamber }\end{array}$ & Array & Institute's QA \\
\hline & & & $\Delta_{N}(\%)$ & \multicolumn{2}{|c|}{ Pass rate (\%) } \\
\hline & & & $\begin{array}{c}\text { Median } \\
(\min -\max )\end{array}$ & $\begin{array}{c}\text { Median } \\
(\min -\max )\end{array}$ & $\begin{array}{c}\text { Median } \\
(\min -\max )\end{array}$ \\
\hline $\begin{array}{l}\text { Simple } \\
\text { IMRT }\end{array}$ & $\operatorname{MLCi}(2)$ & 12 & $\begin{array}{c}-1.5 \\
(-2.8-+0.4)\end{array}$ & $\begin{array}{c}100.0 \\
(98.1-100.0)\end{array}$ & $\begin{array}{c}99.5 \\
(92.4-100.0)\end{array}$ \\
\hline $\begin{array}{l}\text { Simple } \\
\text { VMAT }\end{array}$ & $\operatorname{MLCi}(2)$ & 8 & $\begin{array}{c}-0.6 \\
(-2.5-+1.2)\end{array}$ & $\begin{array}{c}94.0 \\
(75.8-99.6)\end{array}$ & $\begin{array}{c}99.9 \\
(80.3-100.0)\end{array}$ \\
\hline $\begin{array}{l}\text { Complex } \\
\text { IMRT }\end{array}$ & $\operatorname{MLCi}(2)$ & 3 & $\begin{array}{c}-1.4 \\
(-1.4-+0.1)\end{array}$ & $\begin{array}{c}100.0 \\
(99.8-100.0)\end{array}$ & $\begin{array}{c}99.5 \\
(99.3-100.0)\end{array}$ \\
\hline $\begin{array}{l}\text { Complex } \\
\text { IMRT }\end{array}$ & Agility & 10 & $\begin{array}{c}-1.7 \\
(-2.4-+1.3)\end{array}$ & $\begin{array}{c}99.6 \\
(79.0-100.0)\end{array}$ & $\begin{array}{c}99.6 \\
(95.6-100.0)\end{array}$ \\
\hline $\begin{array}{l}\text { Complex } \\
\text { VMAT }\end{array}$ & Agility & 9 & $\begin{array}{c}-2.7 \\
(-3.7--1.4)\end{array}$ & $\begin{array}{c}96.3 \\
(83.3-100.0)\end{array}$ & $\begin{array}{c}98.8 \\
(93.2-100.0)\end{array}$ \\
\hline $\begin{array}{l}\text { Stereotactic } \\
\text { VMAT }\end{array}$ & Agility & 10 & $\begin{array}{c}-2.2 \\
(-2.9--0.9)\end{array}$ & $\begin{array}{c}99.6 \\
(96.9-100.0)\end{array}$ & $\begin{array}{c}100.0 \\
(97.7-100.0)\end{array}$ \\
\hline
\end{tabular}




\subsection{Analysis per linac type - Varian}

In total 28 plans were measured on either type 1 (Clinac) or type 2 (True Beam) linacs.

\subsubsection{Pass/fail agreement between audit and institute QA results}

In Figure 4.5 the agreement between the audit and the institute QA results of the measurements on the Varian linacs are presented.

The majority of the array (22) and ionization chamber (23) measurements were within the tolerances ("Agreement (pass)"). At only one institute the score was ("Agreement (fail)") for both measurement methods. In 5 (array) and 4 (ionization chamber) cases a disagreement was observed between one of the audit measurements and the institute QA.

Four cases for both the array and ionization chamber passed the audit but failed the institute QA. In only one case (complex IMRT plan), the audit failed (array), but the institute QA passed.

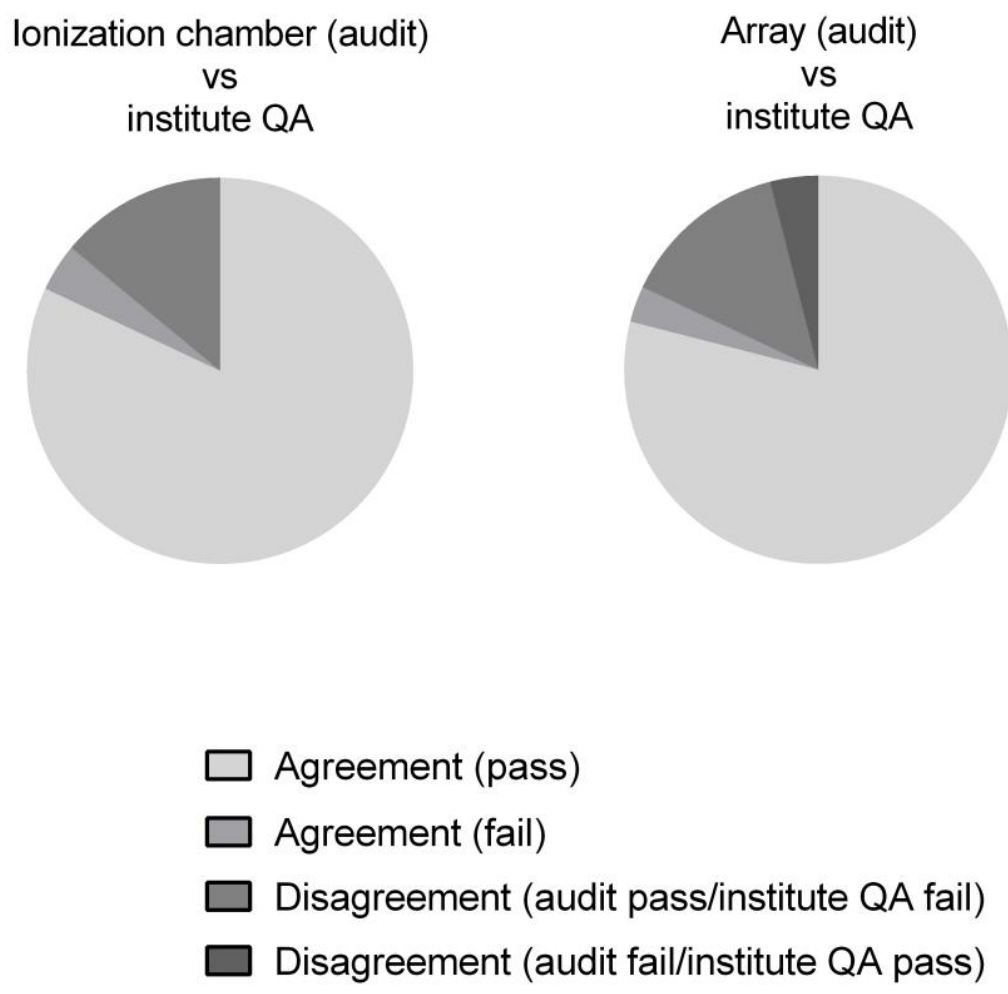

Figure 4.5 Pie-charts showing the results of the agreement between the audit and institute QA measurements, for point and array measurements for all Varian plans. The acceptance criteria were: a gamma pass rate $\geq 95 \%$ ( $5 \%, 1 \mathrm{~mm}$ for the stereotactic plan and $3 \%, 3 \mathrm{~mm}$ for the other plans) for the array measurements; and a relative difference $\pm 3 \%$ for the ionisation chamber measurements 


\subsubsection{Distribution of the audit and institute QA results}

The results of the 28 measurements performed with Varian linacs are summarised in Table 4.4. The average of the median relative difference of the ionization chamber measurements $\left(\Delta_{N}\right)$ for all plan types was $0.8 \pm 0.6 \%$. Compared to the audit results with the Elekta linacs, a wider range was observed for both the IMRT plans. However, this range was in agreement with the institute $Q A$ results.

Table 4.4 Median values of the audit and institute QA for the Varian plans (Clinac and TrueBeam).

\begin{tabular}{|c|c|c|c|c|c|}
\hline Plan type & Linac type & $\begin{array}{c}\text { Number of } \\
\text { measure- } \\
\text { ments }\end{array}$ & $\begin{array}{l}\text { Ionisation } \\
\text { chamber }\end{array}$ & Array & Institute QA \\
\hline & & & $\Delta_{\mathrm{N}}(\%)$ & \multicolumn{2}{|c|}{ Pass rate $(\%)$} \\
\hline & & & $\begin{array}{c}\text { Median } \\
(\min -\max )\end{array}$ & $\begin{array}{c}\text { Median } \\
(\min -\max )\end{array}$ & $\begin{array}{c}\text { Median } \\
(\min -\max )\end{array}$ \\
\hline $\begin{array}{l}\text { Simple } \\
\text { IMRT }\end{array}$ & $\begin{array}{l}\text { Clinac/ } \\
\text { TrueBeam }\end{array}$ & 5 & $\begin{array}{c}-0.2 \\
(-2.1-+0.2)\end{array}$ & $\begin{array}{c}99.1 \\
(93.5-100.0)\end{array}$ & $\begin{array}{c}100.0 \\
(93.8-100.0)\end{array}$ \\
\hline $\begin{array}{l}\text { Simple } \\
\text { VMAT }\end{array}$ & $\begin{array}{l}\text { Clinac/ } \\
\text { TrueBeam }\end{array}$ & 6 & $\begin{array}{c}+1.4 \\
(-0.1-+1.8)\end{array}$ & $\begin{array}{c}100.0 \\
(99.2-100.0)\end{array}$ & $\begin{array}{c}100.0 \\
(92.1-100.0)\end{array}$ \\
\hline $\begin{array}{l}\text { Complex } \\
\text { IMRT }\end{array}$ & $\begin{array}{l}\text { Clinac/ } \\
\text { TrueBeam }\end{array}$ & 5 & $\begin{array}{c}+0.8 \\
(-3.3-+1.9)\end{array}$ & $\begin{array}{c}99.5 \\
(65.8-100.0)\end{array}$ & $\begin{array}{c}97.4 \\
(79.0-100.0)\end{array}$ \\
\hline $\begin{array}{l}\text { Complex } \\
\text { IMRT }\end{array}$ & $\begin{array}{l}\text { Clinac/ } \\
\text { TrueBeam }\end{array}$ & 6 & $\begin{array}{c}+1.2 \\
(-0.9-+1.8)\end{array}$ & $\begin{array}{c}100.0 \\
(99.5-100.0)\end{array}$ & $\begin{array}{c}99.7 \\
(95.0-100.0)\end{array}$ \\
\hline $\begin{array}{l}\text { Complex } \\
\text { VMAT }\end{array}$ & $\begin{array}{l}\text { Clinac/ } \\
\text { TrueBeam }\end{array}$ & 6 & $\begin{array}{c}+0.6 \\
(-0.8-+1.3)\end{array}$ & $\begin{array}{c}100.0 \\
(98.1-100.0)\end{array}$ & $\begin{array}{c}99.7 \\
(94.1-100.0)\end{array}$ \\
\hline $\begin{array}{l}\text { Stereotactic } \\
\text { VMAT }\end{array}$ & $\begin{array}{l}\text { Clinac/ } \\
\text { TrueBeam }\end{array}$ & 5 & $\begin{array}{c}-0.2 \\
(-2.1-+0.2)\end{array}$ & $\begin{array}{c}99.1 \\
(93.5-100.0)\end{array}$ & $\begin{array}{c}100.0 \\
(93.8-100.0)\end{array}$ \\
\hline
\end{tabular}




\section{Discussion}

A national audit on QA systems for treatment plan verification using one set of plans and different measurement methods for all 21 participating centres (22 locations) has proven to be feasible. The main challenges were circumventing problems with DICOM import of RTPlans that was not always possible in the different treatment planning systems and the workload.

\subsection{Feasibility}

The feasibility of a national audit using a single set of treatment plans depends on the possibility to import DICOM data into the institute's TPS, and the available linac types. In the Netherlands, all except three radiotherapy institutes were able to import treatment plans into their TPS. This allowed us to limit the audit to only two sets of treatment plans (i.e. Elekta and Varian treatment plans).

Eclipse, Oncentra and RayStation treatment planning systems have straightforward import options for DICOM plans which originate from other software. Therefore, their users could import the audit treatment plans without much effort. Pinnacle, however, is not capable of importing treatment plans in DICOM format. To accomplish the plan import for these users, the entire plan directory was copied (via the internal Pinnacle backup tool) and adjusted to the Pinnacle version number and linac name of the importing institute. For Monaco and iPlan, no procedure to import the treatment plans was available at the time of the audit. So plans had to be generated for these two TPS by the institute itself based on the same patient CT data sets as the audit plans.

The workload presented another challenge: writing and testing the measurements protocol was time consuming. However, a clear and thoroughly tested measurement protocol turned out to be very helpful for the efficiency of the audit sessions. Furthermore, a clear measurement protocol was necessary to ensure the reproducibility of the measurements.

Finally, given the logistics of the audit, the measurement process was time consuming. The availability of the institute, availability of auditors and, returning the phantom every other week to the owning institute all had to be efficiently coordinated. Since the Netherlands is a relatively small country with few but large radiotherapy institutes, a national audit with a limited number of auditors and one set of equipment was reasonably achievable. 


\subsection{Treatment plans}

In this audit, the same set of treatment plans was delivered in the participating institutes. However, separate plans needed to be created for the different linac types (Elekta MLCi or Agility and Varian) and variations between these plans were unavoidable. For example the number of MUs for the Varian plans was considerably higher than for the Elekta plans (see Table 3.2).

For Elekta linacs, certain machine settings and limits can be chosen by the user, regardless of the TPS that is used. Therefore, during the audit preparation phase, a questionnaire on the employed TPS settings (e.g. maximum leaf speed, minimum and maximum dose rate) chosen by the participants was sent to all Elekta institutes. To ensure that the audit treatment plans could be delivered and the dose could be calculated by each (Elekta) institute, the most conservative settings were chosen (Appendix1D). For Varian users, such freedom in machine settings and limits is not available and the standard settings were used to create the Varian treatment plans.

All treatment plans were generated using $6 \mathrm{MV}$ beams. This photon beam energy was chosen because, at the moment of the audit preparation, it was available for all institutes. The availability of higher beam energies varied too much among participating institutes, and the purpose of the audit was not to investigate beam energy dependence on the QA methods for treatment plan verifications. Moreover, the audit was already challenging for one beam energy. Therefore, as a consequence, higher energy beams were not audited. If the audit for $6 \mathrm{MV}$ is successful it may safely be assumed that higher energies should also comply.

\subsection{Audit results}

The results of this audit show that in general the QA methods for IMRT and VMAT plans in the Netherlands achieve an excellent standard. The median pass rate of the gamma analysis of the $2 \mathrm{D}$ array and film measurements is close to $100 \%$ for all plan types and the average of the median $\Delta_{N}$ for point dose values comprising all plan types is $-1.0 \%$. Furthermore, in the majority of the cases the audit measurements were in agreement with the institute's measurement results (i.e. either both pass or both fail), despite the variation in QA devices in use by the participating institutes. The measurements failing both the audit and institute QA 
were mostly related to the complex plan types. This is in agreement with Lafond et al. who found a lower gamma pass rate for head and neck plans compared to prostate plans [29].

The level of agreement/disagreement between the audit and institute QA results was found to be dependent on audit measurement method. This variation is caused by the characteristics and limitations of the used measurement device (for example the angular dependency of the audit array) in combination with characteristics of the treatment plan.

Moreover, the level of agreement/disagreement between the audit and institute QA results is highly sensitive to the chosen pass/fail limit and acceptance criterion.

In this audit the chosen acceptance criterion for the gamma pass rate is $95 \%$, but if the criterion was $90 \%$, as recommended by the NCS reports 22 and $24[3,4]$, then the level of agreement (both pass) would have increased to 91\%, the level of agreement (both fail) would have decreased to $1 \%$ and the level of disagreement would have reduced to $8 \%$, with $1 \%$ of the cases failing the institute QA and 7\% of the cases failing the audit (the percentages correspond to the average agreement/disagreement among the three measurement methods results).

The degree of agreement/disagreement between the audit results of array and ionization chamber measurements and the institute QA for Elekta or Varian institutes is comparable despite the different set of plans delivered on the two linac vendors. Clark et al. [9] found similar gamma pass rates for Elekta and Varian delivery systems. However, the amount of cases passing the audit but failing the institute QA is higher for the Varian institutes (14\%, average of array and ionization chamber results) than for the Elekta institutes. On the other hand, the percentage of measurements where the audit failed and institute QA passed is larger for the Elekta institutes.

In the array measurement set, a relatively large number of measurements disagreed for the simple and complex VMAT plans (audit failed, institute QA passed), which was most likely due to the measurement device itself. In fact, the response of the 2D array has a directional dependence that influences the outcome of the measurements of these two plans on Elekta linacs. For these plans (simple and complex VMAT), a substantial large number of MU's was delivered at the affected angles (90 and 270 degrees). For the Varian plans, this was not the case. Although the directional dependence of the response was not accounted for during plan optimization, the MU's were more evenly distributed over all angles in the Varian plans resulting in a smaller influence of the directional dependence.

A wider range of results was observed for both simple and complex IMRT plans on Varian machines than that for Elekta linacs. However, this wider range was in agreement with the 
institute QA results. This could be explained by the delivery technique (sliding window), which may be associated with more difficulties in dynamic motion due to, for example, variable leaf speed control and gap separation stability, as opposed with respect to a stepand-shoot delivery $[30,31]$.

Several outliers were observed for the film results, which was more than that of the other measurements methods. For four institutes, a very low gamma pass rate was observed in all film measurements. In these measurements, on average 10 cGy (i.e. 5\% on 200cGy) less dose was recorded by the film when compared to the planned dose. This under-dosage corresponded to a pass rate below $80 \%$ for a $3 \%, 3 \mathrm{~mm}$ gamma criterion. Variations in audit film calibration curves were in the order of $5 \%$, which could explain the outliers. No clear explanation could be found in the audit data set for these differences and further investigations are required to clarify this. However, this is beyond the scope of the audit. A standardized procedure was used to analyse the film measurements in an absolute way. Due to this standardization and based on literature reports [23,32-35] smaller variations ( 1.5\%) were expected in the calibration results. On the other hand, absolute dosimetry with film is known to be more complex than relative film dosimetry.

The QA methods used in this audit were designed to be insensitive to differences in dose calculation properties, for example type and version of TPS, dose algorithm (including dose to water or dose to medium), $\mathrm{HU}$ to electron density conversion and inclusion of the treatment couch. The small variation in $\Delta_{N}$ over all the institutes ionization chamber dose measurements for the different plan types confirms that differences in dose calculation properties do not have a major impact on the QA methods for treatment plan verification. To investigate the influence of the mentioned dose calculation properties on the delivered dose, other tests should be performed. 


\section{Conclusion and recommendations}

This report describes the implementation and results of a national audit on the independent validation of clinically used QA methods. In contrast to other national audits, this audit uses the same set of treatment plans for all institutes. Therefore it enables a comparison of the various QA methods which is not influenced by other steps of the treatment chain such as planning protocol and optimization methods.

The results of the audit show that the QA of IMRT and VMAT plans in the Netherlands achieves an excellent standard. However, a variation persisted between the different audit QA measurement methods. Therefore, the user should be aware of the limitations of each method. We advise to make use of multiple QA measurement systems, in particular to independently validate patient specific QA methods after major modifications in the treatment chain. In addition, participating in independent audits is highly recommended. For other QA recommendations the reader should refer to the NCS reports 22 and 24 [3,4] . 


\section{References}

[1] Budgell G, Berresford J, Trainer M, Bradshaw E, Sharpe P, Williams P. A national dosimetric audit of IMRT. Radiother Oncol 2011;99:246-52.

[2] LoSasso T, Chui C-S, Ling CC. Comprehensive quality assurance for the delivery of intensity modulated radiotherapy with a multileaf collimator used in the dynamic mode. Med Phys 2001;28:2209-19.

[3] Van der Wal E, Wiersma J, Ausma AH, Cuijpers JP, Tomsej M, Bos LJ, et al. NCS Report 22: Code of practice for the quality assurance and control for intensity modulated radiotherapy. NCS 22. Delft: NCS; 2013. doi:10.25030/ncs-022.

[4] Mans A, Schuring D, Arends M, Vugts L, Wolthaus JWH, Lotz H, et al. NCS Report 24: Code of practice for the quality assurance and control for volumetric modulated arc therapy. Delft: 2015. doi:10.25030/ncs-024.

[5] MacKay RI, Staffurth J, Poynter A, Routsis D. UK guidelines for the safe delivery of intensity-modulated radiotherapy. Clin Oncol 2010;22:629-35.

[6] Thwaites D, Scalliet P, Leer JW, Overgaard J. Quality assurance in radiotherapy. European Society for Therapeutic Radiology and Oncology Advisory Report to the Commission of the European Union for the "Europe Against Cancer Programme." Radiother Oncol 1995;35:61-73.

[7] Perik TJ, De Prez LA, Aalbers AHL, Monseux A, Sergent F, Wittkämper FW, et al. NCS Report 23: Audit of high-energy photon beams in Belgian and Dutch radiotherapy departments. Delft: 2013. doi:10.25030/ncs-023.

[8] PTW. Code of practice: OCTAVIUS 4D, how to start Procedure for the implementation and routine use of IMRT 2012:1-19.

[9] Clark CH, Hussein M, Tsang Y, Thomas R, Wilkinson D, Bass G, et al. A multiinstitutional dosimetry audit of rotational intensity-modulated radiotherapy. Radiother Oncol 2014;113:272-8.

[10] http://www.ptw.de/1993.html n.d.

[11] Nederlandse Commissie Voor Stralingsdosimetrie. Code of Practice for the Absorbed Dose Determination in High Energy Photon and Electron Beams. Rep 182008.

[12] Alfonso R, Andreo P, Capote R, Huq MS, Kilby W, Kjäll P, et al. A new formalism for reference dosimetry of small and nonstandard fields. Med Phys 2008;35:5179-86.

[13] Chung E, Bouchard $H$, Seuntjens J. Investigation of three radiation detectors for accurate measurement of absorbed dose in nonstandard fields. Med Phys 2010;37:2404-13.

[14] Rosser KE, Bedford JL. Application of a new dosimetry formalism to volumetric modulated arc therapy (VMAT). Phys Med Biol 2009;54:7045-61.

[15] Van Battum LJ, Huizenga $H$, Verdaasdonk RM, Heukelom $S$. How flatbed scanners upset accurate film dosimetry. Phys Med Biol 2016;61:625-49.

[16] Menegotti L, Delana A, Martignano A. Radiochromic film dosimetry with flatbed scanners: a fast and accurate method for dose calibration and uniformity correction with single film exposure. Med Phys 2008;35:3078-85.

[17] Butson MJ, Cheung T, Yu PKN. Evaluation of the magnitude of EBT Gafchromic film polarization effects. Australas Phys Eng Sci Med 2009;32:21-5.

[18] Butson MJ, Cheung T, Yu PKN. Scanning orientation effects on Gafchromic EBT film dosimetry. Australas Phys Eng Sci Med 2006;29:281-4.

[19] Ranade MK, Li JG, Dubose RS, Kozelka J, Simon WE, Dempsey JF. A prototype quantitative film scanner for radiochromic film dosimetry. Med Phys 2008;35:473-9.

[20] Schoenfeld A a, Poppinga D, Harder D, Doerner K-J, Poppe B. The artefacts of radiochromic film dosimetry with flatbed scanners and their causation by light 
scattering from radiation-induced polymers. Phys Med Biol 2014;59:3575-97.

[21] Shima K, Tateoka K, Saitoh Y, Suzuki J, Yaegashi Y, Fujimoto K, et al. Analysis of Post-exposure Density Growth in Radiochromic Film with Respect to the Radiation Dose. J Radiat Res 2012:301-5.

[22] Chang L, Ho S-Y, Ding H-J, Lee T-F, Chen P-Y. Dependency of EBT2 film calibration curve on postirradiation time. Med Phys 2014;41:21726.

[23] Lewis D, Micke A, Yu X, Chan MF. An efficient protocol for radiochromic film dosimetry combining calibration and measurement in a single scan. Med Phys 2012;39:6339-50.

[24] Lewis D, Chan MF. Correcting lateral response artifacts from flatbed scanners for radiochromic film dosimetry. Med Phys 2015;42:416-29.

[25] Micke A, Lewis DF, Yu X. Multichannel film dosimetry with nonuniformity correction. Med Phys 2011;38:2523-34. doi:10.1118/1.3576105.

[26] Van Esch A, Basta K, Evrard M, Ghislain M, Sergent F, Huyskens DP. The Octavius 1500 2D ion chamber array and its associated phantoms: dosimetric characterization of a new prototype. Med Phys 2014;41:91708.

[27] Van Esch A, Clermont C, Devillers M, lori M, Huyskens DP. On-line quality assurance of rotational radiotherapy treatment delivery by means of a $2 \mathrm{D}$ ion chamber array and the Octavius phantom. Med Phys 2007;34:3825. doi:10.1118/1.2777006.

[28] Hussein M, Adams EJ, Jordan TJ, Clark CH, Nisbet A. A critical evaluation of the PTW 2D-ARRAY seven29 and OCTAVIUS II phantom for IMRT and VMAT verification. J Appl Clin Med Phys 2013;14:4460.

[29] Lafond C, Chiavassa S, Bertaut C, Boussion N, Chapel N, Chapron L, et al. DEMAT: A multi-institutional dosimetry audit of rotational and static intensity-modulated radiotherapy. Phys Medica 2016;32:664-70.

[30] Buckey CR, Stathakis S, Papanikolaou N. The inter- and intrafraction reproducibilities of three common IMRT delivery techniques. Med Phys 2010;37:4854-60.

[31] Alaei P, Higgins PD, Weaver R, Nguyen N. Comparison of dynamic and step-andshoot intensity-modulated radiation therapy planning and delivery. Med Dosim 2004;29:1-6.

[32] Peet SCSC, Wilks R, Kairn T, Trapp JVJ V., Crowe SBSB. Technical Note: Calibrating radiochromic film in beams of uncertain quality. Med Phys 2016;43:5647-52. doi:10.1118/1.4963210.

[33] Tamponi M, Bona R, Poggiu A, Marini P. A new form of the calibration curve in radiochromic dosimetry. Properties and results. Med Phys 2016;43:4435-46.

[34] van Battum LJ, Hoffmans D, Piersma H, Heukelom S. Accurate dosimetry with GafChromic EBT film of a $6 \mathrm{MV}$ photon beam in water: what level is achievable? Med Phys 2008;35:704-16. doi:10.1118/1.2828196.

[35] Wen N, Lu S, Kim J, Qin Y, Huang Y, Zhao B, et al. Precise film dosimetry for stereotactic radiosurgery and stereotactic body radiotherapy quality assurance using Gafchromic $^{\text {TM }}$ EBT3 films. Radiat Oncol 2016;11:1-11. 


\section{Acknowledgements}

The subcommittee would like to thank the NCS for the opportunity given to perform the audit and write this report.

We would also like to thank the institutes for their participation in the audit and for making the accelerators available to perform the measurements. In addition, we thank the local helpers for their contribution to the audit measurements.

Many thanks to Yvonne van Herten and Henry Noordmans for their assistance to the measurements and so reducing the workload of the subcommittee.

Mariet Koopman and Paul Duijvenvoorde, thank you for your help with making of the Varian treatments plans.

We are grateful to Corine van Vliet and Frits Wittkämper of the NKI-AVL for the kind loan of the phantom and the measurement equipment used during the audit.

Last but not least, we would like to thank the external reviewers Jeroen van de Kamer, Leah McDermott and Milan Tomsej for their valuable comments and critical review of the report. 


\section{Appendices}

\section{A. Audit protocol}

\section{THIS PAGE IS PREPARED BEFORE THE AUDIT BY}

NAME

LOCATION

DATE

\section{Preparation of the audit visit}

\section{TO BE PERFORMED Before the visit}

\section{PHONE CALL: planning IMRT ready}

Call the institute to confirm that:

a. The institute has performed its own patient QA for the audit plans, on the linac(s) where the audit will be performed

b. RTDOSE files for the plans, calculated on the Octavius phantom, are available

c. In case of a physical treatment room door (as opposed to a labyrinth), the following connections to the treatment room should be available:

- RS232 (serial) cable or UTP cable

- Low leakage triaxial cable with BNC (bayonet) connector

\section{$\square$ Check and prepare the audit data files on the laptop and copies of paper-datasheets}

a. Make sure a folder called 'C:INCS_IMRT_audit' is available on the laptop

a. Confirm that all previous data has been backed-up to the dropbox folder.

b. Open Excel-workbook called: 'C:INCS_IMRT_auditlMeetsheet NCS Audit.xlsx' (X indicates the version number)

c. Save this workbook as: 'C:INCS_IMRT_auditl<Institute Name>IYYYYMMDD CITY INSTITUTE Meetsheet NCS Audit'. Where YYYYMMDD CITY INSTITUTE is replaced by the year (YYYY), month (MM) and day (DD) of the audit visit, the city of the institute and name of the institute to be visited (e.g. '20100223 Utrecht UMCU')

d. Note: copying the file will not have the same result since it is a read-only file!

- Fill-in ALL required information on the spreadsheet

a. Enter $\underline{A L L}$ required information in the 'MAIN SHEET' of the spreadsheet

b. Save the spreadsheet on the laptop and a backup copy on dropbox

c. Check that the 'auto-save' mode of the spreadsheet is activated

\section{- Check availability and prepare equipment for transportation}
a. Collect all measurement equipment according to the checklist
b. Check if all equipment is in good shape
c. Pack the equipment according to the photos. Package instructions are included in the binder.
d. Make sure these measurement instructions are also printed and included in the box 


\section{Audit visit}

\section{On arrival at the RT-institute}

Institute

Accelerator ID \#1:

Accelerator

ID

\#2:

NCS auditor 1 :

NCS auditor 2 :
Institute representative 1:

Institute representative 2 :

It is advised to divide roles: one person takes the lead in setting up the equipment and fills in these sheets (the other person checks), the second person takes the lead in obtaining all data (the first person checks).

\section{$\square$ Unpack the equipment}

a. Bring all equipment case(s) to the treatment room

b. Unpack equipment from the case(s)

c. Place the equipment at the designated location: (control- or treatment room) see checklist

\section{$\square$ Ask the local representative to prepare the thermometer \& barometer as used locally}

\section{- Switch on the elektrometer (Unidos Webline) \& connect ionisation chamber}

a. Connect the ionisation chamber for point measurements to the Unidos, via the $30 \mathrm{~m}$ extension cable. Leave the ionisation chamber in a safe place where no one will trip over the cable, for example in the equipment case

b. Connect mains power cable to the Unidos Webline

c. Switch on the PTW Unidos Webline using the power switch on the back side

d. Press the power button on the front panel

e. Check if the following settings are applied: RESET; Range: low $271 \mathrm{pA}$; Detector: Audit IMRT; HV: +400CV; Statistics: Manual

\section{S Switch on the Octavius array detector interface}

a. Connect the detector interface to mains.

b. Connect the array to the detector interface.

c. Turn on the detector interface.

d. Connect the Octavius array detector interface to the laptop via the UTP (network) cable, or via RS232 (Serial) cable if UTP is not available. Turn on the laptop. There is no password.

\section{$\square$ Prepare the Excel-workbook}

a. Open on the laptop the Excel-workbook, named: 'C:INCS_IMRT_audit|<Institute Name>|YYYYMMDD CITY INSTITUTE Meetsheet NCS Audit' (see earlier).

b. Fill in the necessary information (e.g. names of auditors, linacs, etc.) 


\section{Array measurements}

\section{Octavius set-up for array measurements}

\section{$\square$ Position and align the Octavius on the treatment table}

a. Place the Octavius bottom (black smiley, with air gap) on the feet.

b. Place the top parts of the Octavius on top.

c. Make sure the phantom is in the center of the table, in the lateral direction

d. Make sure the table rotation is 0 .

e. Insert the detector array. Ensure that it is the right way up.

f. Align the phantom in the linac isocentre, the way a patient would be aligned, according to local guidelines (i.e. use the lasers). If the collimator angle matters for this alignment, make sure it is set at the correct angle.

g. After alignment: Verify that the source surface distance to the top of the Octavius is $84 \mathrm{~cm}$.

Note: the bottom with the black smile is for array measurements, the bottom with the red smile is for film or point measurements. The reason for this is that the black version has an air gap that compensates for the reduced sensitivity of the array when irradiated from below. The red version does not have this air gap.

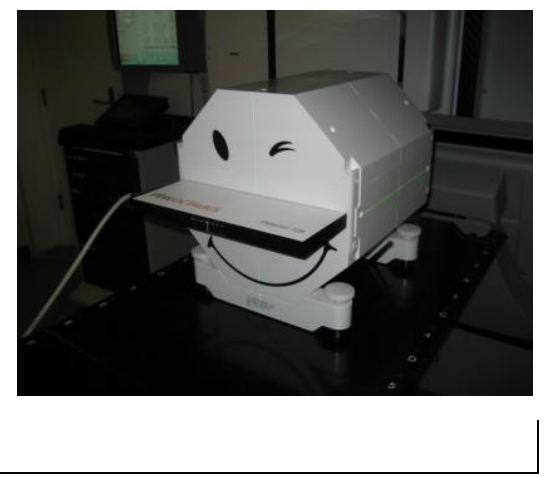

Black smiley!

\section{$\square$ Record the air pressure and temperature in the treatment room}

\begin{tabular}{|l|l|l|}
\hline & Linac \#1 & Linac \#2 \\
\hline Pressure (hPa) & & \\
\hline Temperature (degrees C) & & \\
\hline Time (hh:mm) & & \\
\hline
\end{tabular}




\section{$\square$ Prepare Verisoft for measurement.}

a. Verify that the detector interface is connected to the detector array, and is on.

b. Start Verisoft on the laptop.

c. In Verisoft, go to (Tools)->(Measurement Options), and verify that the settings under "Devices In Use" are as shown in the screenshot.

d. In the same screen, under "Connections", hit the "Search..." button to automatically find the detector interface. If the detector interface was found, hit "OK" to proceed.

e. $\quad$ Go to (File)-> (Data Set A) -> (Measure...)

f. Verify that measurement parameters are as shown in the screenshot. Any corrections will be applied during analysis, we want to measure with $\mathrm{k}=\mathrm{k}_{\mathrm{TP}}{ }^{*} \mathrm{~K}_{\text {User }}{ }^{*} \mathrm{k}_{\text {Energy }}=1.000$. Hit "OK".

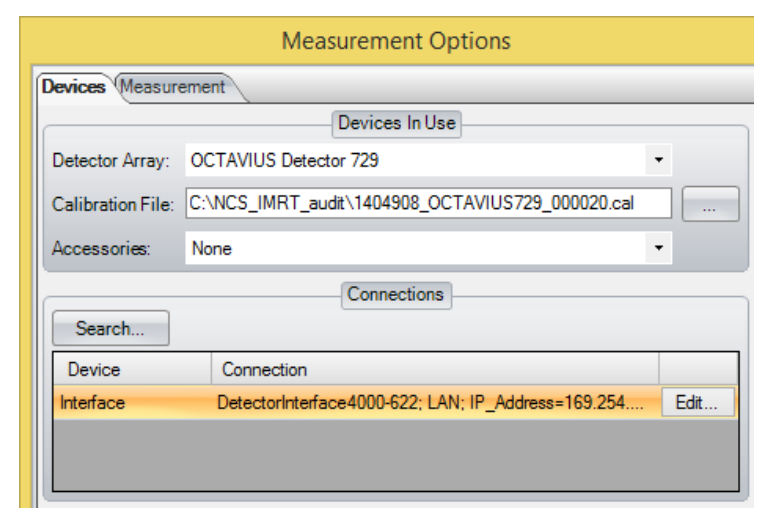

g. You will be prompted to perform a zero measurement.

h. Wait until the zero measurement is finished.

\section{Pre-irradiate the detector array}

a. Verify that there are no people or EBT films in the treatment room.

b. Ensure that the active measurement range is "Low": Go to (Measurement), there should be a checkmark next to "Range Low".

c. Start the measurement (click "Start"), Pre-irradiate the detector array with a $25 \times 25$ field, Collimator angle 0 or 90 degrees, $800 \mathrm{MU}$.

d. Click "Stop", and then "Save". Save the *.mcc file as C:/NCS_IMRT_Audit/<Institute name $>/ 25 \times 25$ before $<$ machinetype $>$.mcc, where $<$ machinetype $>$ is the type of machine being measured, $\mathrm{MLCl}$ or Agility or Clinac or Truebeam.

e. Wait until at least 3 minutes have passed since the pre-irradiation, then perform another Zero measurement (Measurement->Zero). This is to avoid the effect of "ghosting".

\section{$\square$ Perform the measurements}

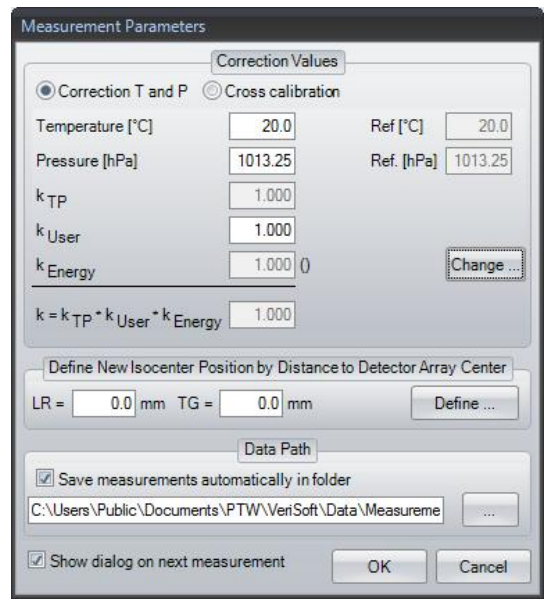

a. Click "Start" and deliver a $10 \times 10$ beam of $200 \mathrm{MU}$.

b. Click "Stop", and then "Save". Save the *.mcc file as C:/NCS_IMRT_Audit/<Institute name $>/ 10 \times 10$ before $<$ machinetype>.mcc

c. Start a new measurement, then fully deliver one of the audit treatment plans (the entire plan, so all beams/arcs).

d. Click "Stop", and then "Save"; Save as C:/NCS_IMRT_Audit/<Institute name $>/<$ plan name $>$.mcc

e. If there are more plans to be delivered at this linac, repeat the previous 2 substeps for the next plan.

f. Finish with another $10 \times 10200 \mathrm{MU}$ beam, save this one as C:/NCS_IMRT_Audit/<Institute name>/10x10 after $<$ machinetype $>$.mcc

\section{$\square$ Record the air pressure and temperature in the treatment room}

\begin{tabular}{|l|l|l|}
\hline & Linac \#1 & \multicolumn{1}{|c|}{ Linac \#2 } \\
\hline Pressure (hPa or mbar) & & \\
\hline Temperature (degrees C) & & \\
\hline Time (hh:mm) & & \\
\hline \multicolumn{2}{|l|}{} \\
\hline
\end{tabular}


Film measurements

Octavius set-up for film measurements

General remarks for film measurements:

- Don't get fingerprints on the film. Hold them by the edges.

- Don't expose the films to light unnecessarily. Keep them in dark envelopes as much as possible.

- Don't leave films in the treatment room!

\section{$\square$ Place a film in the film insert and puncture it}

a. Take a single film from the control room to the treatment room.

b. Insert the film in the film insert.

c. Cover the film with the top part of the film insert

d. Puncture the film through the 5 holes, using the Sharp Object (found inside the film insert, see photo)

e. Put the Sharp Object back in its place

(The 5 holes will be visible on the scan of the film, they define the position of the isocentre on the film)
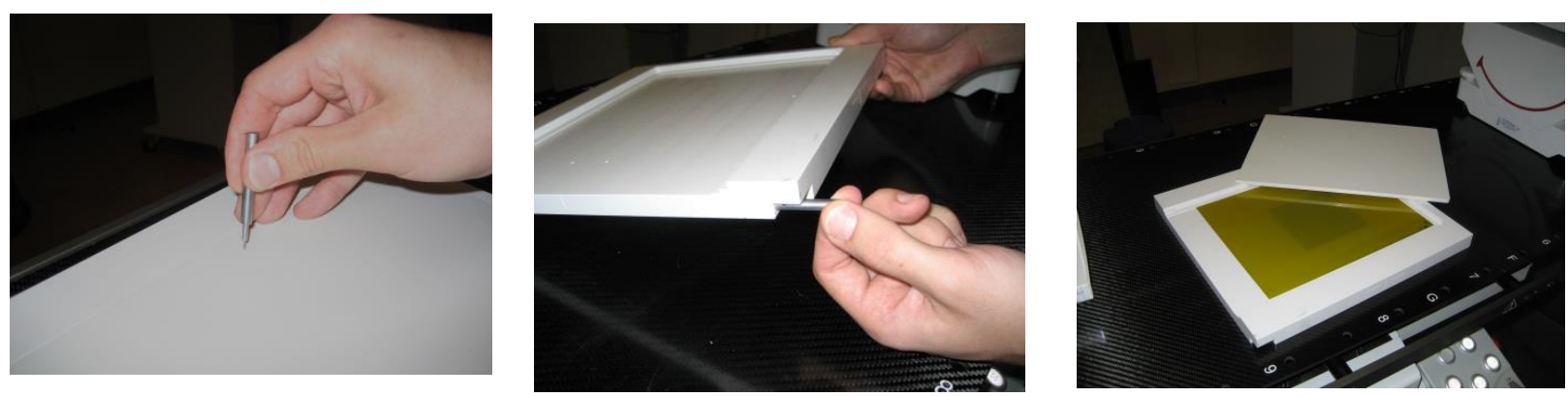

\section{Position and align the Octavius on the treatment table}

a. Place the Octavius bottom (red smiley, without air gap) on the feet.

b. Place the top parts of the Octavius on top.

c. Insert the film insert. Ensure that it is the right way up: The thin "lid" part should be on top.

d. Make sure the phantom is in the centre of the table, in the lateral direction.

e. Align the phantom in the linac isocentre, the way a patient would be aligned, according to local guidelines (i.e. use the lasers). If the collimator angle matters for this alignment, make sure it is set at the correct angle.

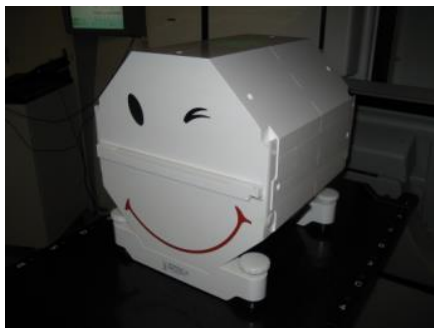

Note: the bottom with the black smile is for array measurements, the bottom with the red smile is for film or point measurements. The reason for this is that the black version has an air gap that compensates for the reduced sensitivity of the array when irradiated from below. The red version does not have this air gap.

\section{RED Smiley}

\section{$\square$ Deliver a treatment plan, mark the film with the required information, and repeat}

a. Before delivering the plan, ensure that the rest of the films are safely in the control room.

b. Deliver the plan.

c. After plan delivery, remove the film, write the institute name, plan name and date on it in a corner with the marker. Put the film in the included envelope.

d. If there are more plans to be measured at this accelerator, repeat the previous steps.

!! Don't ruin a whole stack of films by leaving them in the treatment room during delivery!! 


\section{$\square$ Make a calibration set $(10 \times 10 ; 0,200,400,600 \mathrm{MU})$}

Summary: We need a calibration set of four pieces of film irradiated with a 10x10 field in the Octavius phantom, with the following doses: $0 \mathrm{MU}, 200 \mathrm{MU}, 400 \mathrm{MU}, 600 \mathrm{MU}$. Note: This only needs to be done once per institute, not once per linac, so if this is the second linac, skip this part. In steps:

a. Place a quarter film in the center of the film insert, and place the film insert in the Octavius phantom.

b. Irradiate it with the $10 \times 10$ reference field, $200 \mathrm{MU}$.

c. Mark the quarter film with the institute name and add it to the envelope.

d. Place a quarter film in the center of the film insert, and place the film insert in the Octavius phantom.

e. Irradiate it with the $10 \times 10$ reference field twice, total $400 \mathrm{MU}$.

f. Mark the quarter film with the institute name and add it to the envelope.

g. Place a quarter film in the center of the film insert, and place the film insert in the Octavius phantom.

h. Irradiate it with the $10 \times 10$ reference field three times, total $600 \mathrm{MU}$.

i. Mark the quarter film with the institute name and add it to the envelope.

j. $\quad$ Place an unirradiated quarter film in the envelope.

\section{Point measurements}

\section{$\square$ Position and align the Octavius on the treatment table}

\section{RED Smiley}

a. Verify that you have the red Octavius bottom on the table. Remove the film insert.

b. Insert point measurement inserts. Ensure that it is the right way up. Note the "top" sticker.

c. Insert the PinPoint ionization chamber in the center hole, so that it ends up in the linac isocentre. Make sure it is all the way in.

d. Check the alignment of the Octavius phantom, and adjust if necessary.

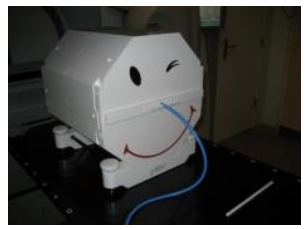

Note: the bottom with the black smile is for array measurements, the bottom with the red smile is for film or point measurements. The reason for this is that the black version has an air gap that compensates for the reduced sensitivity of the array when irradiated from below. The red version does not have this air gap.

\section{Check if the dosimeter has been switched on}

a. Verify that the Unidos Webline has been on for at least 15 minutes.

b. Check if the following settings are applied: RESET; Range: low $271 \mathrm{pA}$; Detector: Audit IMRT; HV: +400V; Statistics: Manual

Note 1: If the settings are not correct change them via the 'SETUP' menu: the main dial serves as a DIAL / BUTTON

Note 2: Measurements will have to be manually started and reset. For plan deliveries, combine the entire plan into a single reading.

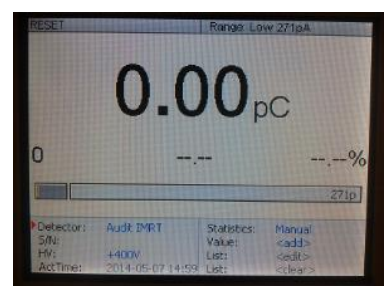

\section{$\square$ Record the air pressure and temperature in the treatment room}

\begin{tabular}{|l|l|l|}
\hline & Linac \#1 & Linac \#2 \\
\hline Pressure (hPa) & & \\
\hline Temperature (degrees C) & & \\
\hline Time (hh:mm) & & \\
\hline
\end{tabular}




\section{Measurements}

\section{$\square$ Pre-irradiate the chamber and perform calibration measurement}

a. Pre-irradiate the ionization chamber with approximately 8 Gy

b. Wait at least 1 minute, then zero the electrometer.

c. Verify that zeroing has properly accounted for leakage: Measure without radiation for 30 seconds, the reading should be $<$ $0.1 \mathrm{pC}$. If the reading exceeds $0.1 \mathrm{pC}$, repeat the zeroing procedure.

\begin{tabular}{|l|l|l|}
\hline Leakage verification & Reading (pC) (linac \#1) & Reading (pC) (linac \#2) \\
\hline 30 seconds, no radiation & & \\
\hline
\end{tabular}

d. Start a measurement on the electrometer, deliver a 10x10 beam, collimator angle as standard in the institute, $200 \mathrm{MU}$, and record the result in the excel file and in the table below. Do this twice.

\section{$\square$ Deliver a treatment plan, record the result, and repeat}

a. Start a measurement on the electrometer

b. Deliver a complete treatment plan (All beams/arcs in a single measurement) and record the reading in the Excel file and on paper.

c. If there are more treatment plans to be measured at this linac, reset the electrometer and repeat.

d. Finish with another 10x10 $200 \mathrm{MU}$ measurement, twice, and record it in the Excel file and on paper.

\begin{tabular}{|l|l|l|}
\hline Plan & Reading (nC) (linac \#1) & Reading (nC) (linac \#2) \\
\hline $10 \times 10 \# 1$ & & \\
\hline $10 \times 10 \# 2$ & & \\
\hline Plan ... & & \\
\hline Plan ... & & \\
\hline Plan ... & & \\
\hline Plan ... & & \\
\hline Plan ... & & \\
\hline $10 \times 10 \# 3$ & & \\
\hline $10 \times 10 \# 4$ & & \\
\hline
\end{tabular}

\section{$\square$ Record the air pressure and temperature in the treatment room}

\begin{tabular}{|l|l|l|}
\hline & Linac \#1 & Linac \#2 \\
\hline Pressure (hPa) & & \\
\hline Temperature (degrees C) & & \\
\hline Time (hh:mm) & & \\
\hline
\end{tabular}




\section{Repeat on the other linac \& clean-up.}

\section{$\square$ Move the equipment to the other linac, if applicable}

a. If measurements are to be performed at another linac, repeat the whole protocol there.

\section{$\square$ Clean up!}
a. Save the excel file.
b. Disconnect the network cable from the Octavius controller, and connect to the hospital's guest wifi.
c. Copy all measurement data to the dropbox folder in the appropriate directory.
d. E-mail all measurement results to the audit team.
e. Pack all equipment in their respective cases. 


\section{List of instruments}

\section{$\underline{\mathrm{CR}}=$ Control Room; TR =Treatment Room}

Checklist instructions:

Upon arrival at the institute, go through the list and mark every piece of equipment that is present in the check IN column. Equipment marked CR should be in the control room during measurements, TR in the treatment room.

When clearing up the equipment, mark the check OUT column.

\begin{tabular}{|c|c|c|c|c|}
\hline Instruments / tools & Manufacturer / type & $\begin{array}{c}\text { check } \\
\text { IN }\end{array}$ & $\begin{array}{l}\text { LOC } \\
\text { IN }\end{array}$ & $\begin{array}{c}\text { check } \\
\text { OUT }\end{array}$ \\
\hline \multicolumn{5}{|c|}{ Octavius phantom } \\
\hline Upper half $(A)$ & PTW & $\square$ & TR & $\square$ \\
\hline Centre pieces (insert slot) $(B, C)$ & PTW & $\square$ & TR & $\square$ \\
\hline Lower half (black) (D) & PTW & $\square$ & TR & $\square$ \\
\hline Lower half (red) (E) & PTW & $\square$ & TR & $\square$ \\
\hline Feet & PTW & $\square$ & TR & $\square$ \\
\hline Film insert & PTW & $\square$ & TR & $\square$ \\
\hline Point measurement insert, 3 pieces & PTW & $\square$ & TR & $\square$ \\
\hline Point measurement filler piece (x9) & PTW & $\square$ & TR & $\square$ \\
\hline \multicolumn{5}{|c|}{729 Array, in carrying case } \\
\hline 729 Array & PTW, T10040, 000020 & $\square$ & TR & $\square$ \\
\hline Detector Interface 4000 & PTW, T16039, 000021 & $\square$ & TR & $\square$ \\
\hline Mains cable & - & $\square$ & TR & $\square$ \\
\hline RS232 (serial) cable, 30m & - & $\square$ & CR & $\square$ \\
\hline UTP (network) cable, 30m & - & $\square$ & CR & $\square$ \\
\hline \multicolumn{5}{|c|}{ Other Measurement equipment } \\
\hline Electrometer PTW Webline T41003 & sn. 0032 & $\square$ & CR & $\square$ \\
\hline Manual of the electrometer PTW Webline & - & $\square$ & CR & $\square$ \\
\hline Mains cable for electrometer & - & $\square$ & CR & $\square$ \\
\hline Ionisation chamber (NCS) & PTW [PinPoint, ser. Nr. 0571] & $\square$ & TR & $\square$ \\
\hline 2 Triax adapters (in PinPoint box) & PTW & $\square$ & TR & $\square$ \\
\hline Extension cable for ionisation chamber (blue) & - & $\square$ & CR & $\square$ \\
\hline Radiochromic films (at least 15 unused) & Gafchromic EBT3 & $\square$ & CR & $\square$ \\
\hline \multicolumn{5}{|c|}{ Computer and accessories } \\
\hline Notebook computer, charger and mouse & [Acer] & $\square$ & CR & $\square$ \\
\hline \multicolumn{5}{|c|}{ Other tools } \\
\hline This protocol & - & $\square$ & CR & $\square$ \\
\hline Marker for writing on EBT3 films & - & $\square$ & CR & $\square$ \\
\hline Envelopes for EBT3 films & - & $\square$ & CR & $\square$ \\
\hline
\end{tabular}




\section{B. Audit preparation manuals}

\section{B1. Elekta manual}

This manual describes the different audit preparation actions and the most crucial steps. The exact methods, applicable to your institute and systems, are not part of this manual. Therefore, we refer you to your local expert for help.

If you have questions or need help, contact your audit contact person.

\section{Loading the audit plans}

1. Download the $\mathrm{CT}$, delineations and plans using the link in the email.

2. Load the Octavius 2D CT and delineation set into the TPS.

a. Patient name and ID can be set according to local guidelines.

3. Load the plans into the TPS.

a. Plan name can be set according to local guidelines, if the relation to the original name is clear.

b. For a description of the plans, see Table 1.

c. Check in Table 2 if you received the correct plans.

i. The set of plans to be audited is only based on available hardware and licenses in your institute.

d. Chose the correct linac, used during the audit, for dose calculation.

e. Do not change the type of linac (=number of leaves).

4. Calculate the dose of each plan using the Octavius 2D CT, as if the Octavius is a clinical patient.

a. Dose grid: $2 \mathrm{~mm}$.

b. Use a density override (ROI = External Octavius).

i. (Electron) Density $=1.016$.

ii. Mass density $=1.04$.

c. Correct for the table top using your protocol.

d. Do not change the order of the beams.

e. Do not change the beam energy (only $6 \mathrm{MV}$ is included in the audit).

f. Number of MU per beam:

i. If your local $\mathrm{MU}$ definition is: $1 \mathrm{MU}=1 \mathrm{cGy}$ at $\mathrm{D}_{\max }$ with $\mathrm{SSD}=100 \mathrm{~cm}$, do not change the amount of MU per beam.

ii. If you have a different MU definition, rescale the MUs per beam. In this case, also contact the audit contact person.

5. Save for each plan:

a. Dose (total dose).

b. The RT-plan file.

c. The dose at the isocenter (in Table 3).

\section{Measurement using local QA system}

6. Perform the QA of each plan according to your local guidelines, as if it were clinical plans.

a. If possible, perform the QA in a coronal plane through the isocenter.

b. Plans can be delivered using your preferable dose rate.

7. Perform also a gamma analysis using: 


\section{Reporting data}

a. Ignore dose below $10 \%$ of the maximum dose.

b. Criteria: $5 \%, 1 \mathrm{~mm}$ (plans $\mathrm{E}$ and $\mathrm{H}$ ) or $3 \%, 3 \mathrm{~mm}$ (other plans).

c. Report the gamma results using Table 3 .

8. Send the data to your audit contact person:

a. Dicom files of the calculated dose (point 5).

i. Dose (total dose).

ii. RT-plan file.

b. Table 3 of this document, completed.

Table 1 NCS Audit IMRT-VMAT plans.

$\begin{array}{lllll}\text { File name } & \text { Plan name } & \text { Linac type } & \text { Technique } & \text { Type of plan } \\ \text { Cal_a } & \text { cal_a } & \text { Agility } & \text { Calibration } & \text { 10x10, 25x25 } \\ \text { Cal_m } & \text { cal_m } & \text { MLCi } & \text { Calibration } & \text { 10x10, 25x25 } \\ \text { Plan_A_sim } & \text { A_sim } & \text { MLCi } & \text { IMRT } & \text { Simple } \\ \text { Plan_B_svm } & \text { B_svm } & \text { MLCi } & \text { VMAT } & \text { Simple } \\ \text { Plan_C_cim } & \text { C_cim } & \text { MLCi } & \text { IMRT } & \text { Complex } \\ \text { Plan_D_cvm } & \text { D_cvm } & \text { MLCi } & \text { VMAT } & \text { Complex } \\ \text { Plan_E_hvm } & \text { E_hvm } & \text { MLCi } & \text { VMAT } & \text { Stereotactic } \\ \text { Plan_F_cia } & \text { F_cia } & \text { Agility } & \text { IMRT } & \text { Complex } \\ \text { Plan_G_cva } & \text { G_cva } & \text { Agility } & \text { VMAT } & \text { Complex } \\ \text { Plan_H_hva } & \text { H_hva } & \text { Agility } & \text { VMAT } & \text { Stereotactic }\end{array}$

Note: Only a selection of plans (a maximum of 5 plans) is audited in each institute (Table 2).

Table 2 Plans to be audited, the type of linac is not adapted. Calibration plans are not given in this table.

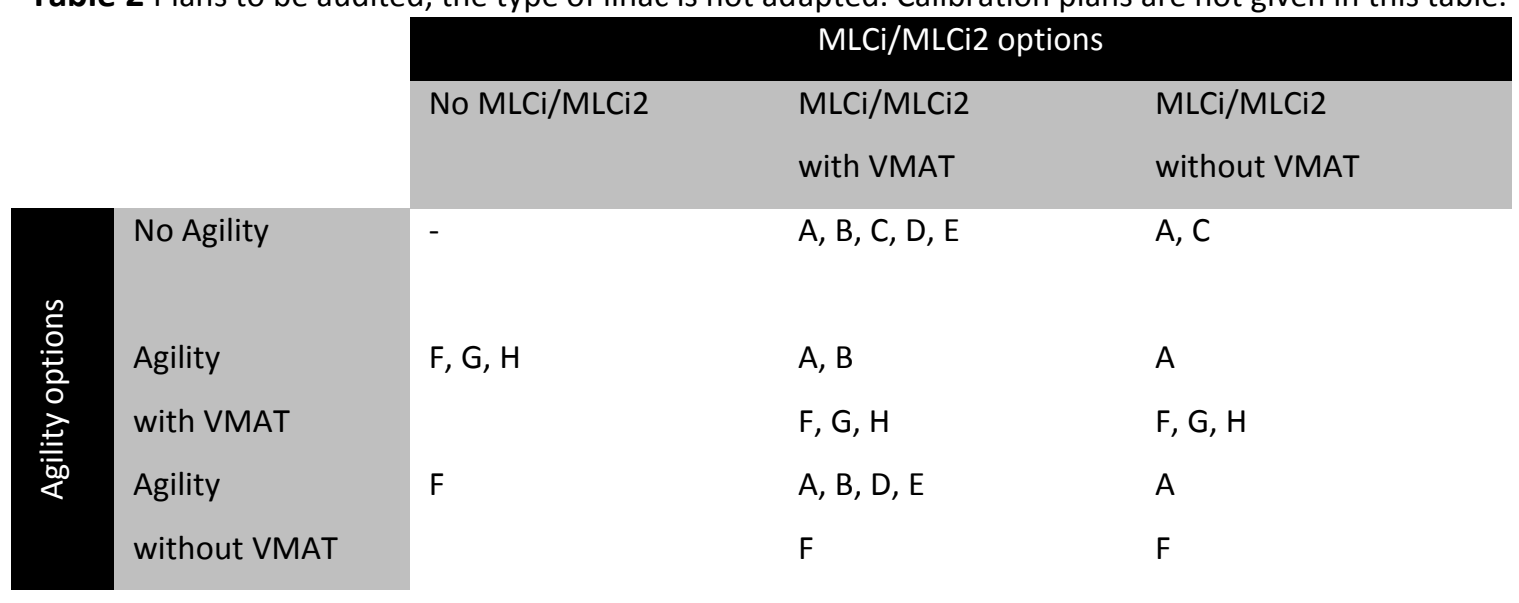


Table 3 Additional information (to be completed by the institute).

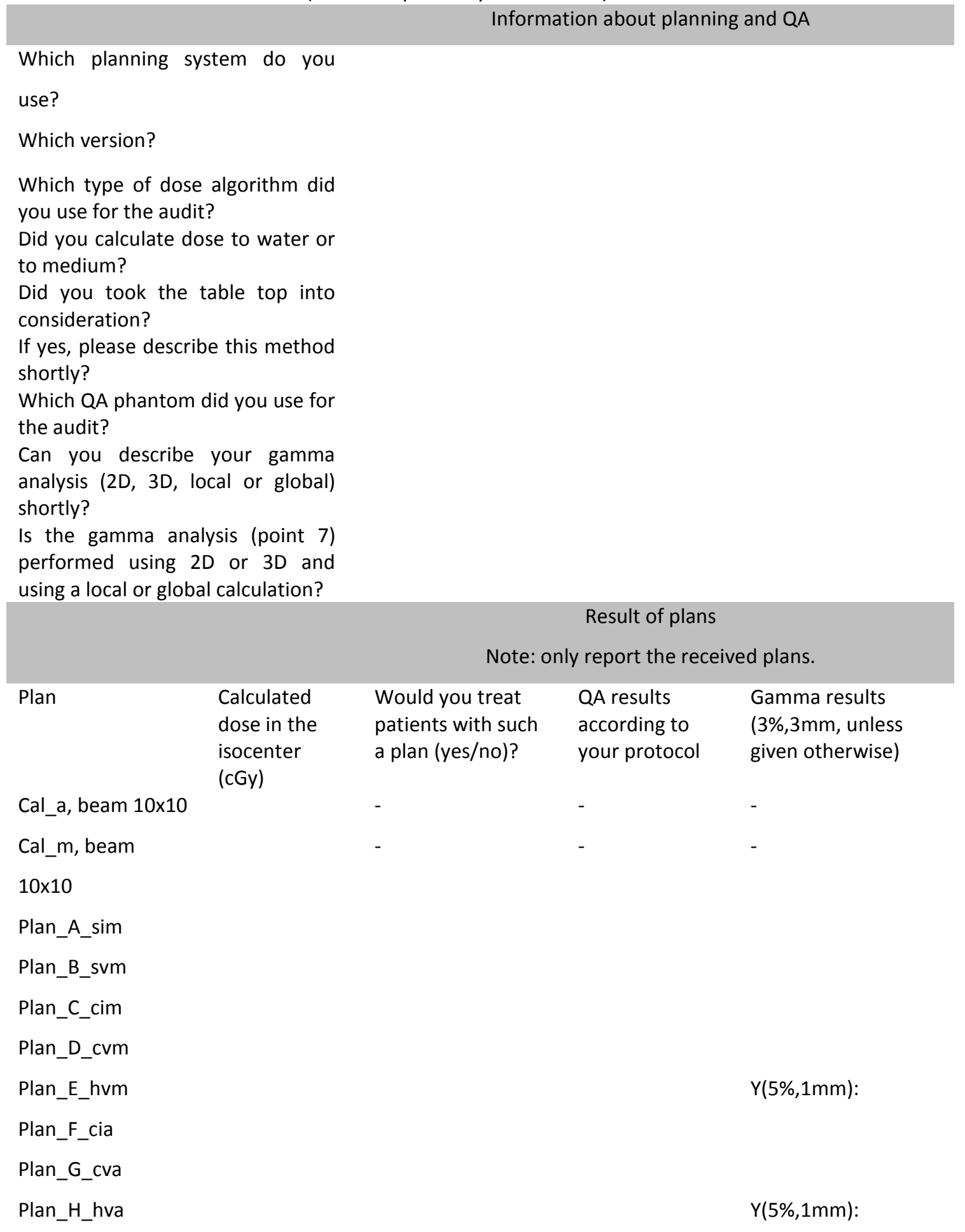

\section{Notes:}




\section{B2. Varian manual}

This manual describes the different audit preparation actions and the most crucial steps. The exact methods, applicable to your institute and systems, are not part of this manual. Therefore, we refer you to your local expert for help.

If you have questions or need help, contact your audit contact person.

\section{Loading the audit plans}

1. Download the $\mathrm{CT}$, delineations and plans using the link in the email.

2. Load the Octavius 2D CT and delineation set into the TPS.

a. Patient name and ID can be set according to local guidelines.

3. Load the plans into the TPS.

a. Plan name can be set according to local guidelines, if the relation to the original name is clear.

b. For a description of the plans, see Table 1.

c. Check in Table 2 if you received the correct plans.

i. The set of plans to be audited is only based on available hardware and licenses in your institute.

d. Chose the correct linac, used during the audit, for dose calculation.

4. Calculate the dose of each plan using the Octavius 2D CT, as if the Octavius is a clinical patient.

a. Dose grid: $2 \mathrm{~mm}$.

b. Use a density override (ROI = External Octavius).

i. (Electron) Density $=1.016$.

ii. Mass density $=1.04$.

c. Correct for the table top using your protocol.

d. Do not change the order of the beams.

e. Do not change the beam energy (only $6 \mathrm{MV}$ is included in the audit).

f. Number of MU per beam:

i. If your local MU definition is: $1 \mathrm{MU}=1 \mathrm{cGy}$ at $\mathrm{D}_{\max }$ with $\mathrm{SSD}=100 \mathrm{~cm}$, do not change the amount of $\mathrm{MU}$ per beam.

ii. If you have a different MU definition, rescale the MUs per beam. In this case, also contact the audit contact person.

5. Save for each plan:

a. Dose (total dose).

b. The RT-plan file.

c. The dose at the isocenter (in Table 3).

Measurement using local QA system

6. Perform the QA of each plan according to your local guidelines, as if it were clinical plans.

a. If possible, perform the QA in a coronal plane through the isocenter.

b. Plans can be delivered using your preferable dose rate.

7. Perform also a gamma analysis using:

a. Ignore dose below $10 \%$ of the maximum dose.

b. Criteria: $5 \%, 1 \mathrm{~mm}$ (plans $\mathrm{E}$ and $\mathrm{H}$ ) or $3 \%, 3 \mathrm{~mm}$ (other plans).

c. Report the gamma results using Table 3.

\section{Reporting data}


8. Send the data to your audit contact person:

c. Dicom files of the calculated dose (point 5).

i. Dose (total dose).

ii. RT-plan file.

d. Table 3 of this document, completed.

Table 1 NCS Audit IMRT-VMAT plans. The prescribed dose percentage is 100\% for all plans.

$\begin{array}{lllll}\text { File name } & \text { Plan name } & \text { Technique } & \text { Type of plan } & \text { Dose/fraction (cGy) } \\ \text { Cal_10 } & \text { Calibration } & \text { Calibration } & 10 \times 10 & 125 \\ \text { Cal_25 } & \text { Calibration } & \text { Calibration } & 25 \times 25 & 595 \\ \text { Plan_A } & \text { IMRT_Simple } & \text { IMRT } & \text { Simple } & 186.5 \\ \text { Plan_B } & \text { VMAT_Simple } & \text { VMAT } & \text { Simple } & 183.4 \\ \text { Plan_C } & \text { IMRT_Complex } & \text { IMRT } & \text { Complex } & 143.9 \\ \text { Plan_D } & \text { VMAT_Complex } & \text { VMAT } & \text { Complex } & 142.4 \\ \text { Plan_E } & \text { VMAT_Hypo } & \text { VMAT } & \text { Stereotactic } & 358.9\end{array}$

Table 2 Plans to be audited, the type of linac is not adapted. Calibration plans are not given in this table.

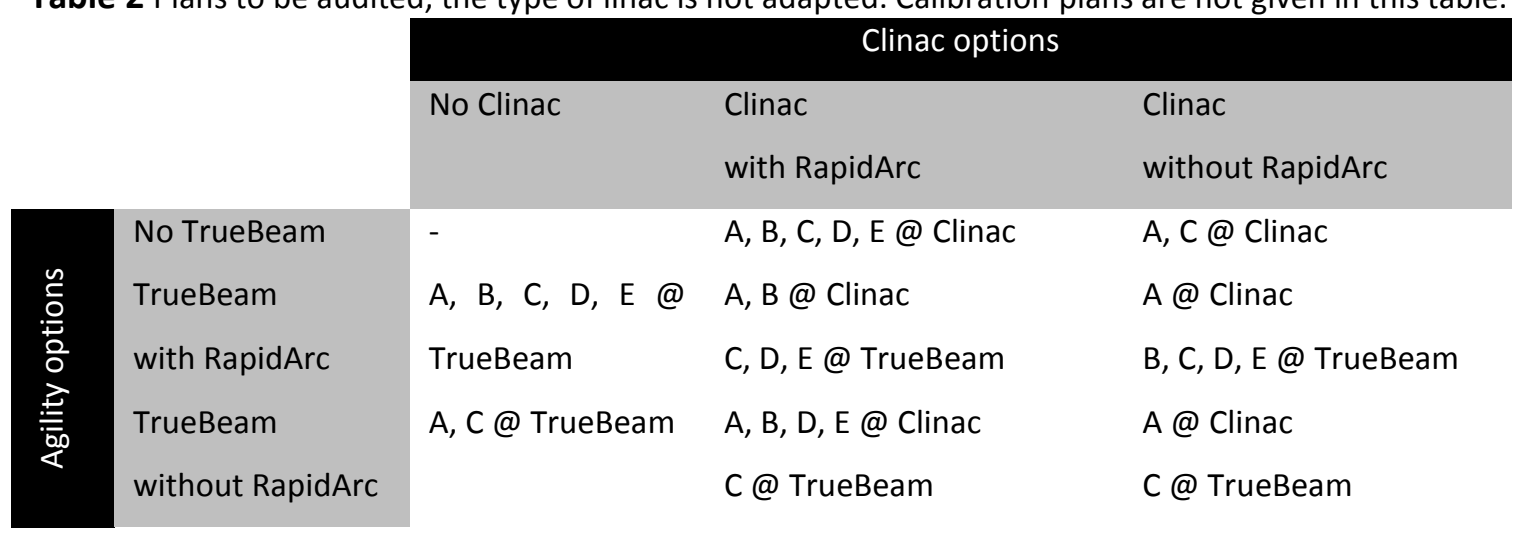


Table 3 Additional information (to be completed by the institute).

Information about planning and QA

Which planning system do you use?

Which version?

Which type of dose algorithm did you use for the audit?

Did you calculate dose to water or to medium?

Did you took the table top into consideration?

If yes, please describe this method shortly?

Which QA phantom did you use for the audit?

Can you describe your gamma analysis (2D, 3D,

local or global) shortly?

Is the gamma analysis (point 7) performed using

2D or 3D and using a local or global calculation?

\begin{tabular}{|c|c|c|c|c|}
\hline \multirow{2}{*}{\multicolumn{2}{|c|}{$\begin{array}{ll}\text { Plan } \quad \begin{array}{l}\text { Calculated } \\
\text { dose in the } \\
\text { isocenter } \\
\text { (cGy) }\end{array}\end{array}$}} & \multirow[b]{2}{*}{$\begin{array}{l}\text { Would you treat } \\
\text { patients with } \\
\text { such a plan } \\
\text { (yes/no)? }\end{array}$} & \multicolumn{2}{|c|}{$\begin{array}{l}\text { Result of plans } \\
\text { Note: only report the received plans. }\end{array}$} \\
\hline & & & $\begin{array}{l}\text { QA results according } \\
\text { to your protocol }\end{array}$ & $\begin{array}{l}\text { Gamma results ( } 3 \%, 3 \mathrm{~mm} \text {, } \\
\text { unless given otherwise) }\end{array}$ \\
\hline $\begin{array}{l}\text { Calibration, } \\
\text { beam } 10 \times 10 \\
\text { Clinac }\end{array}$ & & - & - & - \\
\hline $\begin{array}{l}\text { Calibration, } \\
\text { beam 10x10 } \\
\text { TrueBeam } \\
\text { Plan_A }\end{array}$ & & - & - & - \\
\hline Plan_B & & & & \\
\hline Plan_C & & & & \\
\hline Plan_D & & & & \\
\hline Plan_E & & & & $\mathrm{Y}(5 \%, 1 \mathrm{~mm}):$ \\
\hline
\end{tabular}

\section{Notes:}




\section{Uncertainty budget point measurement}

The uncertainty of $N_{D w}$ follows from the calibration certificate. Since the average dose-perpulse per treatment plan may vary considerably with treatment plans and linac type, an additional contribution for the recombination correction was added to the uncertainty budget. A variation in average dose-per-pulse of a factor 2 (at maximum setting) was assumed over all treatment plans and facilities

The estimated uncertainty of $M_{c o r}$ is based on the long term stability of the PinPoint ionisation chamber and the estimated uncertainty on the $k_{\mathrm{p}, \mathrm{T}}$ measurement.

For the used measured- $k_{Q}$ factor, variations in beam quality of the reference fields between participating institutes are neglected here, but are included as a separate additional uncertainty contribution.

No corrections for the response of the non-standard field effect of the ionisation chamber for each audit treatment plan was made; instead, based on the maximum estimated response effect of $0.9 \%$, an additional contribution of $0.5 \%$ to the uncertainty budget was added.

The relative difference, $\Delta$, between the absorbed dose, as measured in the isocentre by the ionisation chamber, and the planned dose, as provided by the institute, was calculated. This value was multiplied by the ratio of the calculated dose and the measured dose for the $10 \times 10 \mathrm{~cm}^{2}$ field, to determine the reported $\Delta_{\mathrm{N}}$.

Table C. 1 Uncertainty budget for the point dose measurement for an audit treatment plan separated in the Type A (evaluated by statistical analysis) and Type B (evaluated by other means) uncertainties.

\begin{tabular}{|lcc|}
\hline Quantity & $\mathbf{U}_{\mathrm{A}}(\%)$ & $\mathbf{U}_{\mathrm{B}}(\%)$ \\
\hline $\boldsymbol{N}_{D_{w}}$ & & 0.5 \\
Correction for volume recombination & 0.1 \\
$\boldsymbol{M}_{\text {cor }}$ & 0.4 & \\
Long term stability ionisation chamber & & 0.3 \\
$\boldsymbol{k}_{\boldsymbol{Q}}$ & \multicolumn{2}{c|}{0.6} \\
Response change for non-standard fields & \multicolumn{2}{c|}{0.5} \\
Combined standard uncertainty (1 SD) & 1.1 \\
Expanded standard uncertainty (2 SD) & $\mathbf{2 . 1}$ \\
\hline
\end{tabular}




\section{TPS settings for Elekta linacs}

Certain machine settings and limits can be chosen by the user for Elekta linear accelerators, this is regardless of the TPS that is used. Therefore, a questionnaire about the TPS settings (e.g. maximum leaf speed, minimum and maximum dose rate) to be used in the audit treatment plans was sent to all Elekta institutes. To ensure that the audit treatment plans could be delivered and the dose could be calculated in each Elekta institute, the most conservative settings were used to generate the audit plans (Table D.1). Such site-specific settings do not exist for Varian institutes.

Table D.1 The list of the most conservative settings used to generate the Elekta audit plans.

\begin{tabular}{|c|c|c|}
\hline \multirow{2}{*}{$\begin{array}{l}\text { TPS parameter } \\
\text { Machine Information }\end{array}$} & \multicolumn{2}{|c|}{ Settings applied for audit plans } \\
\hline & MLCi & Agility \\
\hline \multicolumn{3}{|l|}{ Jaws } \\
\hline Left/Right: Jaw positions (cm), Min & -12.4 & -12.5 \\
\hline Left/Right: Jaw positions (cm), Max & 20 & 20 \\
\hline Top/Bottom: Jaw positions (cm), Min & 0 & 0 \\
\hline Top/Bottom: Jaw positions (cm), Max & 20 & 20 \\
\hline Decimal Places & 1 & 1 \\
\hline \multicolumn{3}{|l|}{ Delivery } \\
\hline Maximum gantry rotation speed (degree/s) & 5.5 & 5.5 \\
\hline Maximum jaw speed (cm/s) & 1 & 8.5 \\
\hline Maximum MLC leaf speed $(\mathrm{cm} / \mathrm{s})$ & 2 & 3 \\
\hline Limit gantry acceleration? & No & No \\
\hline Maximum gantry MU delivery (MU/degree) & 20 & 20 \\
\hline Minimum gantry MU delivery (MU/degree) & 0.125 & 0.1 \\
\hline Minimum MLC leaf MU delivery (MU/cm) & 0.3125 & 0.3 \\
\hline \multicolumn{3}{|l|}{ MLC information } \\
\hline \multicolumn{3}{|l|}{ General } \\
\hline Leaf Position Decimal places & 1 & 1 \\
\hline \multicolumn{3}{|l|}{ Leaves } \\
\hline Leaf pair number 20: minimum tip position $(\mathrm{cm})$ & -12.4 & -10.5 \\
\hline Leaf pair number 20: maximum tip position (cm) & 20 & 20 \\
\hline Maximum tip difference for all leaves on a side $(\mathrm{cm})$ & 25 & 20 \\
\hline Minimum static leaf gap (cm) & 1 & 0.6 \\
\hline Minimum dynamic leaf gap (cm) & 1.1 & 0.6 \\
\hline \multicolumn{3}{|l|}{ Jaw Dependencies } \\
\hline Maximum leaf/jaw overlap (cm) & 0.5 & $\mathrm{~N} / \mathrm{A}$ \\
\hline Minimum leaf/jaw overlap (cm) & 0.5 & $\bar{N} / \mathrm{A}$ \\
\hline \multicolumn{3}{|l|}{ Beams } \\
\hline Dose rate: minimum & 50 & 50 \\
\hline Dose rate: maximum & 430 & 450 \\
\hline
\end{tabular}

